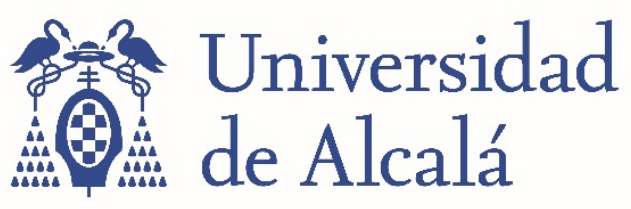

BIBLIOTECA

Document downloaded from the institutional repository of the University of Alcala: http://dspace.uah.es/

This is a postprint version of the following published document:

Perise-Barrios , A.J., Fuentes Paniagua, M.E., Sánchez-Nieves Fernández, J., Serramía Lobera, M.J., Alonso , E., Reguera , R., Gómez Ramírez, R., Mata De La Mata, Francisco J. De La \& Muñoz Fernández, M.A. 2016, "Improved efficiency of ibuprofen by cationic carbosilane dendritic conjugates", Molecular Pharmaceutics, vol. 13, no. 10, pp. 3427-3438.

Available at http://dx.doi.org/10.1021/acs.molpharmaceut.6b00420

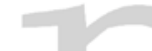
$-1$
(C) 2016 American Chemical Society

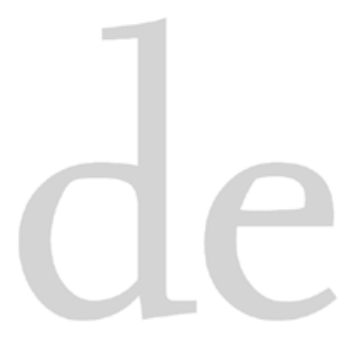

(Article begins on next page)

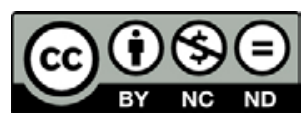

This work is licensed under a

Creative Commons Attribution-NonCommercial-NoDerivatives

4.0 International License. 


\section{IMPROVED EFFICIENCY OF IBUPROFEN BY CATIONIC CARBOSILANE \\ DENDRITIC CONJUGATES}

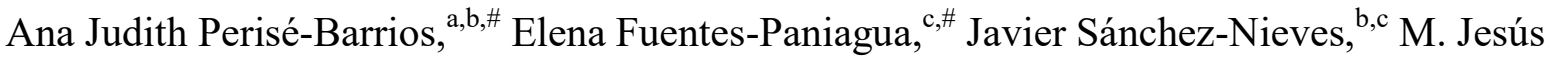
Serramía, ${ }^{a}$ Esther Alonso, ${ }^{a}$ Rosa M. Reguera, ${ }^{\mathrm{d}}$ Rafael Gómez, ${ }^{\text {bcc, }}{ }^{*}$ F. Javier de la Mata, ${ }^{\text {bc* }}$ and M. Ángeles Muñoz-Fernández ${ }^{\mathrm{a}, \mathrm{b}, *}$

${ }^{a}$ Laboratorio Inmuno-Biología Molecular, Hospital General Universitario Gregorio Marañón, Spanish HIV HGM BioBank and Instituto de Investigación Sanitaria Gregorio Marañón, 28007 Madrid, Spain. E-mail: mmunoz.hgugm@gmail.com

${ }^{\mathrm{b}}$ Networking Research Center on Bioengineering, Biomaterials and Nanomedicine (CIBERBBN), Spain.

${ }^{\mathrm{c}}$ Departamento de Química Orgánica y Química Inorgánica, Campus Universitario, Universidad de Alcalá, Alcalá de Henares (Madrid) Spain.E-mail: rafael.gomez@uah.es, javier.delamata@uah.es

${ }^{d}$ Departamento de Ciencias Biomédicas, Universidad de León, Campus de Vegazana s/n, 24071 León, Spain.

\# Authors contributed equally to this work. 
Ibuprofen-conjugated and non-ibuprofen cationic carbosilane dendritic molecules show important antiinflammatory properties on macrophages.
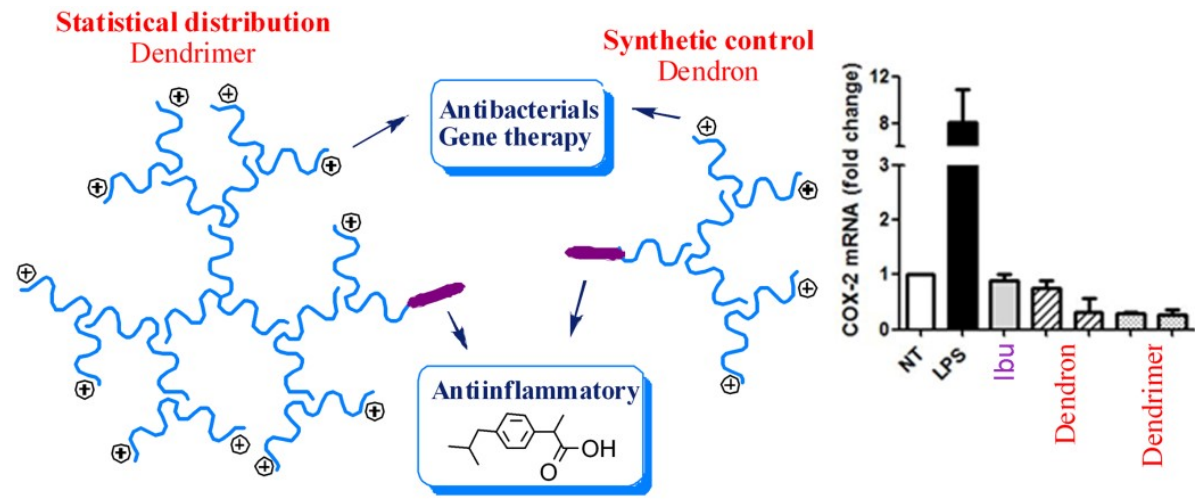


\begin{abstract}
In order to improve the efficiency of the anti-inflammatory drug ibuprofen, cationic carbosilane dendrimers and dendrons with ibuprofen at their periphery or at their focal point, respectively, have been synthesized and the release of the drug was studied using HPLC. Macrophages were used to evaluate the anti-inflammatory effect of the ibuprofen-conjugated dendritic systems and compared with mixtures of non-ibuprofen dendritic systems in the presence of the drug. The cationic ibuprofenconjugated dendron was the compound that showed higher anti-inflammatory properties. It reduces the LPS-induced COX-2 expression, decreases the release of several inflammatory cytokines such as TNF $\alpha$, IL-1 $\beta$, IL-6 and CCL3. These results open new perspectives in the use of these compounds as drug carriers.
\end{abstract}

\title{
KEYWORDS
}

Anti-inflammatory, carbosilane dendrimers, dendrons, ibuprofen, drug release and macrophages. 


\section{INTRODUCTION}

Ibuprofen is a non-steroidal anti-inflammatory drug (NSAID) with antipyretic, analgesic and antibacterial activity, ${ }^{1-3}$ and presents good biocompatibility. ${ }^{4}$ Ibuprofen is a non-selective cyclooxigenase (COX) inhibitor that affects two isoforms, COX-1 (which is constitutively expressed in many cells and tissues) and COX-2 (which is selectively induced by proinflammatory cytokines at the site of inflammation). ${ }^{5}$ The inhibition of COX-2 blocks the prostaglandins biosynthesis, molecules that mediate pathogenic mechanisms as the inflammatory response, and also they are responsible of the pain and fever. ${ }^{6}$ It has been demonstrated that inhibition of COX-2 reverses inflammation and expression of interleukin (IL)- $6 .^{7}$ Therefore, treatments of bacterial infections with ibuprofen hasten the healing process in vaginal infections, cellulites or acne. ${ }^{8-10}$ However, its poor solubility joined to protein binding, diminishes its bioavailability. ${ }^{11}$ This problem can be found in many drugs, ${ }^{12}$ so there is a need to develop new platforms for the transport, protection and sustained release of drugs, minimizing its side effects, as well as avoiding a fluctuation of the therapeutic concentration. ${ }^{13}$ Several systems have been reported for their use as drug carriers such as vesicles, nanoparticles, polymers. ${ }^{14-19}$

Dendrimers are highly branched globular macromolecules which, due to their multivalency, have been widely employed in biomedical applications. ${ }^{20}$ The multivalency of dendrimers, as well as the possibility of employing the cavities in their structure, makes them good candidates for obtaining drug conjugates that can act as carriers, giving solubility to the drugs and protecting them. ${ }^{21-24}$ However, heterofunctionalization of dendrimers usually implies statistical methods of synthesis, and thus, a loss of their characteristic monodispersity. For this reason, other topologies can be used in order to obtain drug conjugates while maintaining a defined structure. Similarly to dendrimers, dendrons are highly branched cone shaped macromolecules, with a focal point, clearly differentiated from dendritic periphery. ${ }^{25}$ This extra reactive moiety can be used to obtain more complex structures by anchoring other structures such as polysaccharides, polymers or nanoparticles. ${ }^{26-29}$ Few examples of drugconjugated dendrons can be found. Bianco et al. reported the synthesis of adamantane based dendrons with ibuprofen moieties at their periphery. These compounds enhanced the anti-inflammatory activity 
of ibuprofen due to dendrimer multivalency. ${ }^{30}$ On the other hand, polyester dendrons have been used to solubilize the hydrophobic dye BODIPY. ${ }^{31}$ Finally, previous works of our research group reported the synthesis of heterofunctionalized cationic carbosilane dendrons with fluorescein. ${ }^{32,33}$ In this case, biodistribution studies on carbosilane dendrimers showed their capability to cross the blood brain barrier, ${ }^{34}$ which is an important obstacle in drug delivery to the brain. Regarding ibuprofen modified dendrimers, PAMAM-ibuprofen dendrimers improve the drug's efficacy by enhancing cellular delivery, which may produce a rapid pharmacological response ${ }^{35}$ depending of the linkage between dendrimer and drug. ${ }^{36}$ Also has been reported that ibuprofen solubility increases by electrostatic interactions with dendrimers or by conjugation through bond formation. ${ }^{37,38}$

The study of the anti-inflammatory effect induced by compounds with ibuprofen requires an appropriate in vitro system, being macrophages the suitable model due to their regulatory capacities in inflammatory responses. The immune system is responsible of maintenance the homeostasis on the human body. To do it properly, detects pathogens and trigger different immune responses involving a wide variety of cells with the aim to eliminate the pathogen. Macrophages are one of the most important cells that regulate and trigger different immune responses with the aim to eliminate pathogens. ${ }^{39}$ To trigger an inflammatory response, macrophages must have a pro-inflammatory phenotype expressing cytokines such as IL-1 $\beta$, IL-6 and TNF- $\alpha^{40-42}$ and exhibiting microbicidal functions. These cells are defined as M1-polarized macrophages. Once they have accomplished their objective, and therefore an immune response has been successfully activated, macrophages eliminate microorganisms by phagocytosis and also restore tissue homeostasis. Macrophages that are acting in the resolution of the inflammation are M2-polarized macrophages that present an anti-inflammatory phenotype, defined by the production of anti-inflammatory and regulatory cytokines such as IL-10 and TGF- $\beta{ }^{43,44}$

Herein we deal with the synthesis of cationic carbosilane dendritic macromolecules with one ibuprofen unit bonded to them with the aim to evaluate the effect of this drug in these systems. Previous studies warned about the importance of evaluating the inflammatory effect of molecules with 
a future biological application, ${ }^{45}$ as this factor will cause significant problems inducing in a noncontrolled way the immune system responses. Moreover, it is well known that some dendritic derivatives failed in biological studies performed in vitro due to the inflammatory effect over cells. ${ }^{46}$ On the other hand, since cationic carbosilane dendritic molecules have been proved useful as siRNA delivery vectors against HIV $^{47-49}$ and as microbicides, ${ }^{50,51}$ and several types of dendrimers have shown antiinflammatory activities, ${ }^{49,} 52-54$ is very interesting to evaluate them as ibuprofen-carriers, and also to find whether these structures could help to minimize any inflammatory process, effect that could accelerate the healing processes. Finally, the analysis of activity of these compounds would also allow verifying their pharmacological action and thus, their potential use as drug delivery systems or codrugs. 55

For this purpose, a comparative evaluation of cytokine release by treated macrophages has been carried out for cationic carbosilane dendritic molecules with and without an ibuprofen bonded to them. Macrophages have been treated with cationic dendritic systems (dendrimer or dendron), with combinations of free ibuprofen with cationic carbosilane dendrimers/dendrons, and with ibuprofenconjugated cationic systems (dendrimer or dendron). The ibuprofen moiety has been bonded through a labile ester bond, using the carboxylic group present in the drug, in order to favor the release of the integral drug in optimum conditions. ${ }^{56}$

\section{MATERIAL AND METHODS}

\section{General considerations}

All reactions were carried out under inert atmosphere and solvents were purified from appropriate drying agents when necessary. NMR spectra were recorded on a Varian Unity VXR-300 (300.13 $\left({ }^{1} \mathrm{H}\right)$, $\left.75.47\left({ }^{13} \mathrm{C}\right) \mathrm{MHz}\right)$ or on a Bruker AV400 (400.13 (1H), $\left.100.60\left({ }^{13} \mathrm{C}\right), 40.56\left({ }^{15} \mathrm{~N}\right), 79.49\left({ }^{29} \mathrm{Si}\right) \mathrm{MHz}\right)$. Chemical shifts $(\delta)$ are given in ppm. ${ }^{1} \mathrm{H}$ and ${ }^{13} \mathrm{C}$ resonances were measured relative to internal deuterated solvent peaks considering TMS $=0 \mathrm{ppm}$, meanwhile ${ }^{29} \mathrm{Si}$ resonances were measured relative to external MeNO and TMS, respectively. When necessary, assignment of resonances was 
done from HSQC, HMBC, COSY and TOCSY NMR experiments. Elemental analyses were performed on a LECO CHNS-932. Mass Spectra were obtained from a Thermo Scientific TSQ Quantum LC-MS and an Agilent 6210. Thiol-ene reactions were carried out employing a HPK 125 W mercury lamp from Heraeus-Noblelight with maximum energy at $365 \mathrm{~nm}$, in normal glassware under an inert atmosphere. Compounds $\mathrm{HS}\left(\mathrm{CH}_{2}\right)_{2} \mathrm{NH}_{2} \cdot \mathrm{HCl}, \mathrm{HS}\left(\mathrm{CH}_{2}\right)_{2} \mathrm{NMe}_{2} \cdot \mathrm{HCl}, 2,2$ '-dimethoxy-2phenylacetophenone (DMPA), MeI, S-(+)-ibuprofen, $\mathrm{K}_{2} \mathrm{CO}_{3}, \mathrm{Na}_{2} \mathrm{CO}_{3}, \mathrm{NaOH}$, porcine liver esterease (PLE, Aldrich), ethylcarbodiimide (EDCI), 4-dimethylaminopyridine (DMAP) were obtained from commercial sources. Compounds $\mathrm{G}_{2} \mathrm{O}_{3} \mathrm{~V}_{12},{ }^{57} \quad \mathrm{BrG}_{\mathrm{n}} \mathrm{V}_{\mathrm{m}},{ }^{58} \quad \mathrm{G}_{2} \mathrm{O}_{3}\left(\mathrm{NMe}_{3}{ }^{+}\right)_{12} \quad(\mathbf{1 8})^{57}$ and $\mathrm{HOC}_{2} \mathrm{H}_{4} \mathrm{OG}_{3}\left(\mathrm{NMe}_{3}{ }^{+}\right)_{8}(\mathbf{1 9})^{51}$ were synthesized as previously reported.

\section{HPLC}

Release of ibuprofen was monitored at $154 \mathrm{~nm}$ (10.39 min) using an Agilent Technologies 1200 series with an Eclipse XDB-C18 $5 \mu \mathrm{M}$ column (Agilent) of $4.6 \times 150 \mathrm{~mm}$, with a flux of $1 \mathrm{ml} / \mathrm{min}$ and a mixture of acetic acid:acetonitrile (50:50) as eluent. In order to quantify the amount of ibuprofen, a calibration curve was performed between 15 and $275 \mathrm{mg} / \mathrm{l}$ of drug (detection limit is $5 \mathrm{mg} / \mathrm{ml}$, quantification limit is $15 \mathrm{mg} / \mathrm{ml}$ ). Then, PLE ( 1 unit: $1 \mu \mathrm{mol}$ ester) were added to dendritic aqueous solutions of a known concentration close to $200 \mathrm{mg} / \mathrm{l}$ and incubated. Aliquots were taken at several times in order to monitor the release of ibuprofen. The aliquots were measured directly from the reaction systems without quenching. In order to check that complete release was possible, an excess of PLE was added to the incubated mixtures and drug release was HPLC monitored.

\section{Synthesis of compounds}

General procedures for the synthesis of compounds are described and a selected number of compounds are collected below. The complete list of compounds is collected in the supplementary information.

\section{Procedure 1: thiol-ene addition}

To a dendrimer or dendron solution (THF:MeOH, 1:2) decorated with vinyl groups, 1.05 equivalents of the thiol derivative were added and 2,2'-dimethoxy-2-phenylacetophenone (DMPA) 
was used as initiator in a $5 \% \mathrm{~mol}$ regarding the double bonds. The mixture was deoxygenated and irradiated for $1.5 \mathrm{~h}\left(\lambda_{\text {máx }}=364 \mathrm{~nm}\right)$. After this time, another $5 \% \mathrm{~mol}$ of DMPA was added and the mixture was again deoxygenated and irradiated for $1.5 \mathrm{~h}$. Afterwards volatiles were removed under vacuum and compounds were purified using nanofiltration (Stirred Ultrafiltration Cells from Millipore) or dialysis in water, using membranes with the adequate molecular weight cut off according to the size of the corresponding dendritic systems (see experimental in supplementary information).

In the case in which two different thiols were employed, the first derivative was added in a stoichiometric amount, according to the number of vinyl groups to be modified, and irradiated for 30 minutes in the same conditions than those described above. Then, the rest of the periphery was functionalized using the procedure above described after addition of the corresponding amount of the second thiol.

\section{Procedure 2: neutralization of $-\mathrm{NMe}_{2} \cdot \mathrm{HCl}$}

To a dendritic solution in a mixture of $\mathrm{H}_{2} \mathrm{O} / \mathrm{CHCl}_{3}(1: 1)$, an aqueous solution of $\mathrm{NaOH}$ or $\mathrm{Na}_{2} \mathrm{CO}_{3}$ (1.3 equivalents regarding the $-\mathrm{NMe}_{2} \cdot \mathrm{HCl}$ groups) was added. The reaction mixture was stirred for 15 minutes at room temperature and then, the organic phase was separated and dried with $\mathrm{Na}_{2} \mathrm{SO}_{4}$. Finally, volatiles were removed under vacuum to obtain the desired products.

\section{Procedure 3: quaternization of amines with MeI}

In a schlenk, the desired amount of $\mathrm{G}_{2} \mathrm{O}_{3}(\mathrm{OH})\left(\mathrm{NMe}_{2}\right)_{11}$ or $\mathrm{IbuCO}_{2} \mathrm{G}_{\mathrm{n}}\left(\mathrm{NMe}_{2}\right)_{\mathrm{m}}$ was solved in a mixture of dry $\mathrm{Et}_{2} \mathrm{O}$ and THF (10:1). Afterwards, excess MeI (1.05 equivalents per amine) was added at room temperature. The mixture was stirred at room temperature overnight under inert atmosphere and afterwards volatiles were removed under vacuum and products were washed with hexane.

\section{Procedure 4: Introduction of ibuprofen at the focal point of dendrons}

To a DMF solution of $\mathrm{BrG}_{\mathrm{n}} \mathrm{V}_{\mathrm{m}}$ the stoichiometric amount of $\mathrm{S}-(+)$-ibuprofen was added in presence of two equivalents of $\mathrm{K}_{2} \mathrm{CO}_{3}, 0.1$ equivalents of 18-crown-6 and catalytic amounts of NaI. After heating for one night at $80^{\circ} \mathrm{C}$ in a sealed ampoule, volatiles were removed under vaccum and 
$\mathrm{Et}_{2} \mathrm{O} / \mathrm{NH}_{4} \mathrm{Cl}$ (ac) extraction was performed. The organic phase was dried under $\mathrm{MgSO}_{4}$ and solvent removed under vacuum to obtain the desired compounds as oils.

$\mathbf{G}_{2} \mathbf{O}_{3}(\mathbf{O H})\left(\mathbf{N M e}_{2} \cdot \mathbf{H C l}\right)_{11}$ (2). Procedure 1. Step 1: $\mathrm{G}_{2} \mathrm{O}_{3} \mathrm{~V}_{12} \quad(0.575 \mathrm{~g}, 0.46 \mathrm{mmol})$, 2mercaptoethanol (0.03 $\mathrm{ml}, \quad 0.46 \mathrm{mmol})$ and DMPA (0.012 g, $0.04 \mathrm{mmol})$; Step 2: 2(dimethylamino)ethanethiol hydrochloride $(0.788 \mathrm{~g}, 5.28 \mathrm{mmol})$ and DMPA (0.129 $\mathrm{g}, 0.50 \mathrm{mmol})$. Compound 2 was obtained as a white powder $(0.127 \mathrm{~g}, 82 \%)$.

${ }^{1} \mathrm{H}$ NMR (DMSO-d $): \delta-0.07$ (s, $9 \mathrm{H}, \mathrm{OC}_{4} \mathrm{H}_{8} \mathrm{SiMe}$ ), 0.01 (s, $\left.18 \mathrm{H}, \mathrm{SiMeC} \mathrm{H}_{2} \mathrm{H}_{4} \mathrm{~S}\right), 0.58(\mathrm{~m}, 30 \mathrm{H}$, $\mathrm{OCH}_{2} \mathrm{CH}_{2} \mathrm{CH}_{2} \mathrm{CH}_{2} \mathrm{SiCH}_{2}$ and $\left.\mathrm{CH}_{2} \mathrm{SiC}_{2} \mathrm{H}_{3}\right), 0.85$ (m, $\left.24 \mathrm{H}, \quad \mathrm{SiCH}_{2} \mathrm{CH}_{2} \mathrm{~S}\right), 1.32$ (m, $18 \mathrm{H}$, $\mathrm{OCH}_{2} \mathrm{CH}_{2} \mathrm{CH}_{2} \mathrm{CH}_{2} \mathrm{Si}$ and $\mathrm{SiCH}_{2} \mathrm{CH}_{2} \mathrm{CH}_{2} \mathrm{Si}$ ), 1.67 (m, $6 \mathrm{H}, \mathrm{OCH}_{2} \mathrm{CH}_{2} \mathrm{CH}_{2}$ ), 2.57 (m, $24 \mathrm{H}$, $\left.\mathrm{SiCH}_{2} \mathrm{CH}_{2} \mathrm{~S}\right), 2.73\left(\mathrm{~s}, 68 \mathrm{H}, \mathrm{SCH}_{2} \mathrm{CH}_{2} \mathrm{O}\right.$ and.$\left.-\mathrm{NMe}_{2} \mathrm{H}^{+}\right), 2.87\left(\mathrm{~m}, 22 \mathrm{H}, \mathrm{SCH}_{2} \mathrm{CH}_{2} \mathrm{~N}^{+}\right.$and $\left.\mathrm{SCH}_{2} \mathrm{CH}_{2} \mathrm{O}\right), 3.19\left(\mathrm{~m}, 20 \mathrm{H}, \mathrm{CH}_{2} \mathrm{~N}^{+}\right), 3.50\left(\mathrm{~m}, 2 \mathrm{H}, \mathrm{SCH}_{2} \mathrm{CH}_{2} \mathrm{O}\right), 3.88\left(\mathrm{~m}, 6 \mathrm{H}, \mathrm{OCH}_{2} \mathrm{CH}_{2} \mathrm{CH}_{2}\right), 6.01$ (s, $\left.3 \mathrm{H}, \mathrm{C}_{6} H_{4}\right) .{ }^{13} \mathrm{C}\left\{{ }^{1} \mathrm{H}\right\}$ NMR $\left(\mathrm{DMSO}_{-} \mathrm{d}_{6}\right): \delta$-5.7 and -5.4 (SiMe), $12.7\left(\mathrm{OCH}_{2} \mathrm{CH}_{2} \mathrm{CH}_{2} \mathrm{CH}_{2} \mathrm{Si}\right), 13.5$ $\left(\mathrm{SiCH}_{2} \mathrm{CH}_{2} \mathrm{SC}_{2} \mathrm{H}_{4} \mathrm{~N}^{+}\right), 13.8\left(\mathrm{SiCH}_{2} \mathrm{CH}_{2} \mathrm{SC}_{2} \mathrm{H}_{4} \mathrm{O}\right), 16.9-17.6\left(\mathrm{SiCH}_{2} \mathrm{CH}_{2} \mathrm{CH}_{2} \mathrm{Si}\right), 19.5\left(\mathrm{OCH}_{2} \mathrm{CH}_{2} \mathrm{CH}_{2}\right)$, $24.3\left(\mathrm{SiCH}_{2} \mathrm{CH}_{2} \mathrm{SC}_{2} \mathrm{H}_{4} \mathrm{~N}^{+}\right), 26.0\left(\mathrm{SCH}_{2} \mathrm{CH}_{2} \mathrm{~N}^{+}\right), 26.4\left(\mathrm{SiCH}_{2} \mathrm{CH}_{2} \mathrm{SC}_{2} \mathrm{H}_{4} \mathrm{O}\right), 32.1\left(\mathrm{OCH}_{2} \mathrm{CH}_{2} \mathrm{CH}_{2}\right), 33.2$ $\left(\mathrm{SCH}_{2} \mathrm{CH}_{2} \mathrm{O}\right), 41.4\left(-\mathrm{NMe}_{2} \mathrm{H}^{+}\right), 55.3\left(\mathrm{CH}_{2} \mathrm{~N}^{+}\right), 60.4\left(\mathrm{SCH}_{2} \mathrm{CH}_{2} \mathrm{O}\right), 66.6\left(\mathrm{OCH}_{2} \mathrm{CH}_{2} \mathrm{CH}_{2}\right), 93.1\left(\mathrm{C}_{6} \mathrm{H}_{4}\right.$, $C$-H), $160.0\left(\mathrm{C}_{6} \mathrm{H}_{4}, C-\mathrm{O}\right)$. Anal. Calc. $\mathrm{C}_{115} \mathrm{H}_{264} \mathrm{Cl}_{11} \mathrm{~N}_{11} \mathrm{O}_{4} \mathrm{~S}_{12} \mathrm{Si}_{9}(2892.93 \mathrm{~g} / \mathrm{mol}): \mathrm{C}, 47.75 ; \mathrm{H}, 9.20 ; \mathrm{N}$, 5.33; S, 13.30; Obt.: C, 48.21; H, 8.71; N, 5.69; S, 12.01.

$\mathbf{G}_{\mathbf{2}} \mathbf{O}_{\mathbf{3}}(\mathbf{O H})\left(\mathrm{NMe}_{2}\right)_{11}$ (3). Procedure 2. Data: 2 (0.600 g, $\left.0.21 \mathrm{mmol}\right)$ and $\mathrm{NaOH}(0.100 \mathrm{~g}, 2.50$ mmol). Compound 3 was obtained as yellowish oil (0.418 g, $81 \%)$.

${ }^{1} \mathrm{H}$ NMR $\left(\mathrm{CDCl}_{3}\right): \delta-0.08\left(\mathrm{~s}, 9 \mathrm{H}, \mathrm{OC}_{4} \mathrm{H}_{8} \mathrm{SiMe}\right), 0.00\left(\mathrm{~s}, 18 \mathrm{H}, \mathrm{Si} M e \mathrm{C}_{2} \mathrm{H}_{4} \mathrm{~S}\right), 0.54(\mathrm{~m}, 30 \mathrm{H}$, $\mathrm{OCH}_{2} \mathrm{CH}_{2} \mathrm{CH}_{2} \mathrm{CH}_{2} \mathrm{SiCH}_{2}$ and $\left.\mathrm{CH}_{2} \mathrm{SiC}_{2} \mathrm{H}_{3}\right), 0.87\left(\mathrm{t}, \mathrm{J}_{\mathrm{a}}=8.7 \mathrm{~Hz}, 24 \mathrm{H}, \mathrm{SiCH}_{2} \mathrm{CH}_{2} \mathrm{~S}\right), 1.28(\mathrm{~m}, 12 \mathrm{H}$, $\left.\mathrm{SiCH}_{2} \mathrm{CH}_{2} \mathrm{CH}_{2} \mathrm{Si}\right), 1.41\left(\mathrm{~m}, 6 \mathrm{H}, \mathrm{OCH}_{2} \mathrm{CH}_{2} \mathrm{CH}_{2} \mathrm{CH}_{2} \mathrm{Si}\right), 1.75\left(\mathrm{~m}, 6 \mathrm{H}, \mathrm{OCH}_{2} \mathrm{CH}_{2} \mathrm{CH}_{2}\right), 2.24(\mathrm{~s}, 66 \mathrm{H}$, $\mathrm{NMe}$ ), $2.48\left(\mathrm{~m}, 22 \mathrm{H}, \mathrm{SCH}_{2} \mathrm{CH}_{2} \mathrm{~N}\right), 2.51\left(\mathrm{~m}, 24 \mathrm{H}, \mathrm{SiCH}_{2} \mathrm{CH}_{2} \mathrm{~S}\right), 2.57\left(\mathrm{~m}, 22 \mathrm{H}, \mathrm{SCH}_{2} \mathrm{CH}_{2} \mathrm{~N}\right), 2.70$ (m, $\left.2 \mathrm{H}, \mathrm{SCH}_{2} \mathrm{CH}_{2} \mathrm{O}\right), 3.68\left(\mathrm{~m}, 2 \mathrm{H}, \mathrm{SCH}_{2} \mathrm{CH}_{2} \mathrm{O}\right), 3.88$ (m, $\left.6 \mathrm{H}, \mathrm{OCH}_{2} \mathrm{CH}_{2} \mathrm{CH}_{2}\right), 6.04\left(\mathrm{~s}, 3 \mathrm{H}, \mathrm{C}_{6} H_{4}\right)$. ${ }^{13} \mathrm{C}\left\{{ }^{1} \mathrm{H}\right\}$ NMR $\left(\mathrm{CDCl}_{3}\right): \delta$-5.2 and -5.1 $\left(\mathrm{SiCH}_{3}\right), 13.8\left(\mathrm{OCH}_{2} \mathrm{CH}_{2} \mathrm{CH}_{2} \mathrm{CH}_{2} \mathrm{Si}\right), 14.7\left(\mathrm{SiCH}_{2} \mathrm{CH}_{2} \mathrm{~S}\right)$, 18.3, 18.4 and $18.6\left(\mathrm{SiCH}_{2} \mathrm{CH}_{2} \mathrm{CH}_{2} \mathrm{Si}\right), 20.6\left(\mathrm{OCH}_{2} \mathrm{CH}_{2} \mathrm{CH}_{2}\right), 27.7\left(\mathrm{SiCH}_{2} \mathrm{CH}_{2} \mathrm{~S}\right), 29.7\left(\mathrm{SCH}_{2} \mathrm{CH}_{2} \mathrm{~N}\right)$, 
$33.3\left(\mathrm{OCH}_{2} \mathrm{CH}_{2} \mathrm{CH}_{2}\right), 35.0\left(\mathrm{SCH}_{2} \mathrm{CH}_{2} \mathrm{O}\right), 45.3\left(-\mathrm{NMe}_{2}\right), 59.2\left(\mathrm{CH}_{2} \mathrm{~N}^{+}\right), 60.7\left(\mathrm{SCH}_{2} \mathrm{CH}_{2} \mathrm{O}\right), 67.7$ $\left(\mathrm{OCH}_{2} \mathrm{CH}_{2} \mathrm{CH}_{2}\right), 93.7\left(\mathrm{C}_{6} \mathrm{H}_{4}, C-\mathrm{H}\right), 160.9\left(\mathrm{C}_{6} \mathrm{H}_{4}, C-\mathrm{O}\right)$. Anal. Calc. $\mathrm{C}_{115} \mathrm{H}_{253} \mathrm{~N}_{11} \mathrm{O}_{4} \mathrm{~S}_{12} \mathrm{Si}_{9}(2491.86$ g/mol): C, 55.43; H, 10.23; N, 6.18; S, 11.99. Obt.: C, 55.81; H, 10.23; N, 6.69; S, 11.89.

$\mathbf{G}_{\mathbf{2}} \mathrm{O}_{\mathbf{3}}(\mathbf{O C O I b u})\left(\mathrm{NMe}_{2}\right)_{11}$ (4). A dry DMF solution of $\mathbf{3}(0.114 \mathrm{~g}, 0.04 \mathrm{mmol}), 2$ equivalents of S(+)-ibuprofeno (0.019 g, $0.09 \mathrm{mmol})$ and 4 equivalents of DMAP (0.047 g, $0.18 \mathrm{mmol})$ was stirred overnight at room temperature under inert atmosphere in a valved ampoule. Then, 4 equivalents of EDCI $(0.035 \mathrm{~g}, 0.18 \mathrm{mmol})$ were added and the solution was heated at $60{ }^{\circ} \mathrm{C}$ for 2 days. Afterwards, volatiles were removed, THF was added and after filtering, a size exclusion chromatographic column (Bio-Beads SX-1, Bio-Rad) was performed in THF obtaining 4as yellowish oil (0.115 g, 93 \%).

${ }^{1} \mathrm{H}$ NMR $\left(\mathrm{CDCl}_{3}\right): \delta-0.07\left(\mathrm{~s}, 9 \mathrm{H}, \mathrm{OC}_{4} \mathrm{H}_{8} \mathrm{SiMe}\right),-0.01\left(\mathrm{~s}, 18 \mathrm{H}, \mathrm{SiMeC}_{2} \mathrm{H}_{4} \mathrm{~S}\right), 0.55(\mathrm{~m}, 30 \mathrm{H}$, $\left.\mathrm{OCH}_{2} \mathrm{CH}_{2} \mathrm{CH}_{2} \mathrm{CH}_{2} \mathrm{SiCH}_{2} \mathrm{CH}_{2} \mathrm{CH}_{2} \mathrm{Si}\right), 0.86\left(\mathrm{~m}, 30 \mathrm{H}, \mathrm{SiCH}_{2} \mathrm{CH}_{2} \mathrm{~S}\right.$ and $\left.\mathrm{CH}\left(\mathrm{CH}_{3}\right)_{2}\right), 1.26(\mathrm{~m}, 21 \mathrm{H}$, $\left.\mathrm{SiCH}_{2} \mathrm{CH}_{2} \mathrm{CH}_{2} \mathrm{Si}\right), 1.42\left(\mathrm{~m}, 6 \mathrm{H}, \mathrm{OCH}_{2} \mathrm{CH}_{2} \mathrm{CH}_{2} \mathrm{CH}_{2} \mathrm{Si}\right), 1.45$ (d, J = 7.2 Hz, $\left.3 \mathrm{H}, \mathrm{CH}\left(\mathrm{CH}_{3}\right) \mathrm{CO}\right), 1.76$ (m, $7 \mathrm{H}, \mathrm{OCH}_{2} \mathrm{CH}_{2} \mathrm{CH}_{2}$ y $\left.\left.\mathrm{CH}\left(\mathrm{CH}_{3}\right)_{2}\right), 2.21(\mathrm{~s}, 66 \mathrm{H},-\mathrm{NMe})_{2}\right), 2.42\left(\mathrm{~m}, 2 \mathrm{H}, \mathrm{CH}_{2} \mathrm{Ar}\right), 2.47(\mathrm{~m}, 22 \mathrm{H}$, $\left.\mathrm{SCH}_{2} \mathrm{CH}_{2} \mathrm{~N}\right), 2.50\left(\mathrm{~m}, 24 \mathrm{H}, \mathrm{SiCH}_{2} \mathrm{CH}_{2} \mathrm{~S}\right), 2.57\left(\mathrm{~m}, 22 \mathrm{H}, \mathrm{SCH}_{2} \mathrm{CH}_{2} \mathrm{~N}\right), 2.70\left(\mathrm{~m}, 2 \mathrm{H}, \mathrm{SCH}_{2} \mathrm{CH}_{2} \mathrm{O}\right)$, $3.88\left(\mathrm{~m}, 6 \mathrm{H}, \mathrm{OCH}_{2} \mathrm{CH}_{2} \mathrm{CH}_{2}\right), 4.17\left(\mathrm{~m}, 2 \mathrm{H}, \mathrm{CH}_{2} \mathrm{OCO}\right), 6.05\left(\mathrm{~s}, 3 \mathrm{H}, \mathrm{C}_{6} H_{4}\right), 7.10$ and $7.16(\mathrm{~m}, 4 \mathrm{H}$, Ar, C- $H) .{ }^{13} \mathrm{C}\left\{{ }^{1} \mathrm{H}\right\}$ NMR $\left(\mathrm{CDCl}_{3}\right): \delta$-5.3 and -5.1 $\left(\mathrm{SiCH}_{3}\right), 13.5\left(\mathrm{OCH}_{2} \mathrm{CH}_{2} \mathrm{CH}_{2} \mathrm{CH}_{2} \mathrm{Si}\right), 14.6$ ( $\left.\mathrm{SiCH}_{2} \mathrm{CH}_{2} \mathrm{~S}\right)$, 18.3-18.6 $\left(\mathrm{SiCH}_{2} \mathrm{CH}_{2} \mathrm{CH}_{2} \mathrm{Si}\right.$ and $\left.\left.\left(\mathrm{CH}_{3}\right) \mathrm{CH}\right), 20.5\left(\mathrm{OCH}_{2} \mathrm{CH}_{2} \mathrm{CH}_{2}\right), 22.4\left(\mathrm{CH}_{(} \mathrm{CH}_{3}\right)_{2}\right)$, $27.6\left(\mathrm{SiCH}_{2} \mathrm{CH}_{2} \mathrm{~S}\right), 29.5\left(\mathrm{SCH}_{2} \mathrm{CH}_{2} \mathrm{~N}\right), 30.1\left(\mathrm{CH}\left(\mathrm{CH}_{3}\right)_{2}\right), 33.2\left(\mathrm{OCH}_{2} \mathrm{CH}_{2} \mathrm{CH}_{2}\right), 34.9\left(\mathrm{SCH}_{2} \mathrm{CH}_{2} \mathrm{O}\right)$, 45.0 (CHCO), $45.2(-\mathrm{NMe}), 45.4\left(\mathrm{CH}_{2} \mathrm{Ar}\right), 59.1\left(\mathrm{CH}_{2} \mathrm{~N}^{+}\right), 63.6\left(\mathrm{SCH}_{2} \mathrm{CH}_{2} \mathrm{O}\right), 67.9\left(\mathrm{OCH}_{2} \mathrm{CH}_{2} \mathrm{CH}_{2}\right)$, 93.6 $\left(\mathrm{C}_{6} \mathrm{H}_{4}, C-\mathrm{H}\right), 127.1$ and $129.3\left(\mathrm{C}_{6} \mathrm{H}_{4(\mathrm{Ibu})}, C-\mathrm{H}\right), 137.5$ y $140.5\left(\mathrm{C}_{6} \mathrm{H}_{4(\mathrm{Ibu})}, C-\mathrm{H}\right), 160.9\left(\mathrm{C}_{6} \mathrm{H}_{4}, C-\right.$ O), $174.5(C=\mathrm{O})$. Anal. Calc. $\mathrm{C}_{128} \mathrm{H}_{269} \mathrm{~N}_{11} \mathrm{O}_{5} \mathrm{~S}_{12} \mathrm{Si}_{9}(2680.13 \mathrm{~g} / \mathrm{mol}): \mathrm{C}, 57.36 ; \mathrm{H}, 10.12 ; \mathrm{N}, 5.75 ; \mathrm{S}$, 14.36; Obt.: C, 57.61; H, 9.84; N, 5.29; S, 14.06.

$\mathbf{G}_{\mathbf{2}} \mathrm{O}_{3}(\mathbf{O C O I b u})\left(\mathrm{NMe}_{3} \mathrm{I}\right)_{\mathbf{1 1}}$ (5). Procedure 3. Data: $4(0.028 \mathrm{~g}, 0.01 \mathrm{mmol})$ and $\mathrm{MeI}(0.01 \mathrm{ml}, 0.13$ mmol). Compound 5 was obtained as a yellowish powder $(0.028 \mathrm{~g}, 65 \%)$.

${ }^{1} \mathrm{H}$ NMR (DMSO-d $\left.)_{6}\right): \delta-0.06\left(\mathrm{~s}, 9 \mathrm{H}, \mathrm{OC}_{4} \mathrm{H}_{8} \mathrm{SiMe}\right), 0.04\left(\mathrm{~s}, 18 \mathrm{H}, \mathrm{SiMeC} \mathrm{H}_{2} \mathrm{H}\right.$ ), $0.56(\mathrm{~m}, 30 \mathrm{H}$, $\mathrm{OCH}_{2} \mathrm{CH}_{2} \mathrm{CH}_{2} \mathrm{CH}_{2} \mathrm{SiCH}_{2}$ and $\left.\mathrm{CH}_{2} \mathrm{SiC}_{2} \mathrm{H}_{3}\right), 0.84\left(\mathrm{~m}, 30 \mathrm{H}, \mathrm{SiCH}_{2} \mathrm{CH}_{2} \mathrm{~S}\right.$ and $\left.\mathrm{CH}\left(\mathrm{CH}_{3}\right)_{2}\right), 1.34$ (m, 21 
$\mathrm{H}, \mathrm{SiCH}_{2} \mathrm{CH}_{2} \mathrm{CH}_{2} \mathrm{Si}$ and $\left.\mathrm{CHCH}_{3}\right), 1.43\left(\mathrm{~m}, 6 \mathrm{H}, \mathrm{OCH}_{2} \mathrm{CH}_{2} \mathrm{CH}_{2} \mathrm{CH}_{2} \mathrm{Si}\right), 1.75\left(\mathrm{~m}, 7 \mathrm{H}, \mathrm{OCH}_{2} \mathrm{CH}_{2} \mathrm{CH}_{2}\right.$ and $\left.\mathrm{CH}\left(\mathrm{CH}_{3}\right)_{2}\right), 2.63\left(\mathrm{~m}, 24 \mathrm{H}, \mathrm{SiCH}_{2} \mathrm{CH}_{2} \mathrm{~S}\right), 2.90\left(\mathrm{~m}, 22 \mathrm{H}, \mathrm{SCH}_{2} \mathrm{CH}_{2} \mathrm{~N}^{+}\right), 3.10\left(\mathrm{~s}, 99 \mathrm{H},-\mathrm{NMe}_{3}{ }^{+}\right)$, $3.53\left(\mathrm{~m}, 22 \mathrm{H}, \mathrm{SCH}_{2} \mathrm{CH}_{2} \mathrm{~N}^{+}\right), 3.86\left(\mathrm{~m}, 6 \mathrm{H}, \mathrm{OCH}_{2} \mathrm{CH}_{2} \mathrm{CH}_{2}\right), 4.13\left(\mathrm{~m}, 2 \mathrm{H}, \mathrm{CH}_{2} \mathrm{OCO}\right), 6.01(\mathrm{~s}, 3 \mathrm{H}$, $\left.\mathrm{C}_{6} H_{4}\right), 7.08$ and $7.15(\mathrm{~m}, 4 \mathrm{H}, \mathrm{Ar}, \mathrm{C}-H) .{ }^{13} \mathrm{C}\left\{{ }^{1} \mathrm{H}\right\}$ NMR $\left(\mathrm{DMSO}_{-} \mathrm{d}_{6}\right): \delta \quad-5.7\left(\mathrm{SiCH}_{3}\right), 13.7$ ( $\left.\mathrm{SiCH}_{2} \mathrm{CH}_{2} \mathrm{~S}\right), 17.4\left(\mathrm{SiCH}_{2} \mathrm{CH}_{2} \mathrm{CH}_{2} \mathrm{Si}\right), 19.6\left(\mathrm{OCH}_{2} \mathrm{CH}_{2} \mathrm{CH}_{2}\right), 21.7\left(\mathrm{CH}\left(\mathrm{CH}_{3}\right)_{2}\right), 23.1\left(\mathrm{SiCH}_{2} \mathrm{CH}_{2} \mathrm{~S}\right)$, $26.4\left(\mathrm{SCH}_{2} \mathrm{CH}_{2} \mathrm{~N}\right), 29.1\left(\mathrm{OCH}_{2} \mathrm{CH}_{2} \mathrm{CH}_{2}\right), 43.7\left(\mathrm{CH}_{2} \mathrm{Ar}\right), 47.8\left(-\mathrm{NMe}_{3}^{+}\right), 64.0\left(\mathrm{CH}_{2} \mathrm{~N}^{+}\right), 126.6$ and $128.7\left(\mathrm{C}_{6} \mathrm{H}_{4(\mathrm{lbu})}, C-\mathrm{H}\right)$. Anal. Calc. $\mathrm{C}_{139} \mathrm{H}_{302} \mathrm{I}_{11} \mathrm{~N}_{11} \mathrm{O}_{5} \mathrm{~S}_{12} \mathrm{Si}_{9}(4241.45 \mathrm{~g} / \mathrm{mol}): \mathrm{C}, 39.36 ; \mathrm{H}, 7.18 ; \mathrm{N}$, 3.63; S, 9.07; Obt.: C, 38.80; H, 7.17; N, 3.16; S, 9.36.

IbuCO $\mathbf{O}_{2} \mathbf{G}_{3} \mathbf{V}_{\mathbf{8}}$ (8). Procedure 4. Data: $\mathrm{BrG}_{3} \mathrm{~V}_{8}(0.627 \mathrm{~g}, 0.69 \mathrm{mmol}), \mathrm{IbuCO}_{2} \mathrm{H}(0.142 \mathrm{~g}, 0.69$ $\mathrm{mmol}), \mathrm{K}_{2} \mathrm{CO}_{3}(0.191 \mathrm{~g}, 1.38 \mathrm{mmol})$ and $18 \mathrm{C} 6$ (0.017 g, $\left.0.06 \mathrm{mmol}\right)$. Yellow oil (0.669 g, 94 \%).

${ }^{1} \mathrm{H} \mathrm{NMR}\left(\mathrm{CDCl}_{3}\right): \delta-0.12$ and $-0.11(\mathrm{~s}, 9 \mathrm{H}, \mathrm{SiMe}), 0.11\left(\mathrm{~s}, 12 \mathrm{H}, \mathrm{SiMeC}_{2} \mathrm{H}_{3}\right), 0.42\left(\mathrm{t}, \mathrm{J}_{\mathrm{a}}=8.4 \mathrm{~Hz}, 2\right.$ $\left.\mathrm{H}, \mathrm{OCH}_{2} \mathrm{CH}_{2} \mathrm{CH}_{2} \mathrm{CH}_{2} \mathrm{Si}\right), 0.53\left(\mathrm{~m}, 16 \mathrm{H}, \mathrm{SiCH}_{2} \mathrm{CH}_{2} \mathrm{CH}_{2} \mathrm{Si}\right.$, overlapped), $0.68\left(\mathrm{t}, \mathrm{J}_{\mathrm{b}}=8.1 \mathrm{H}\right.$, $\left.\mathrm{CH}_{2} \mathrm{SiC}_{2} \mathrm{H}_{3}\right), 0.87\left(\mathrm{~d}, \mathrm{~J}_{\mathrm{c}}=6.6 \mathrm{~Hz}, 6 \mathrm{H},\left(\mathrm{CH}_{3}\right)_{2} \mathrm{CH}\right), 1.32\left(\mathrm{~m}, 14 \mathrm{H}, \mathrm{OCH}_{2} \mathrm{CH}_{2} \mathrm{CH}_{2} \mathrm{CH}_{2} \mathrm{Si}\right.$ and $\left.\mathrm{SiCH}_{2} \mathrm{CH}_{2} \mathrm{CH}_{2} \mathrm{Si}\right), 1.46\left(\mathrm{~d}, \mathrm{~J}_{\mathrm{d}}=7.2 \mathrm{~Hz}, 3 \mathrm{H}, \mathrm{CH}(\mathrm{CH})_{3} \mathrm{CO}\right), 1.57\left(\mathrm{~m}, 2 \mathrm{H}, \mathrm{OCH}_{2} \mathrm{CH}_{2}\right), 1.80(\mathrm{~m}, 1 \mathrm{H}$, $\left.\left(\mathrm{CH}_{3}\right)_{2} \mathrm{CH}\right), 2.42\left(\mathrm{~d}, \mathrm{~J}_{\mathrm{e}}=6.9 \mathrm{~Hz}, 2 \mathrm{H}, \mathrm{CH}_{2} \mathrm{Ar}\right), 3.64\left(\mathrm{q}, \mathrm{J}_{\mathrm{e}}=6.9 \mathrm{~Hz}, 1 \mathrm{H}, \mathrm{CHCO}\right), 4.03\left(\mathrm{t}, \mathrm{J}_{\mathrm{f}}=6.4 \mathrm{~Hz}, 2\right.$ $\left.\mathrm{H}, \mathrm{OCH}_{2}\right), 5.68$ and $6.06\left(\mathrm{~m}, 24 \mathrm{H}, \mathrm{SiCHCH}_{2}\right), 7.07$ and $7.16(\mathrm{~m}, 4 \mathrm{H}, \mathrm{Ar}, \mathrm{C}-H) .{ }^{13} \mathrm{C}\left\{{ }^{1} \mathrm{H}\right\} \mathrm{NMR}$ $\left(\mathrm{CDCl}_{3}\right): \delta$-5.2 and -5.0 $\left(\mathrm{SiCH}_{3}\right), 13.6\left(\mathrm{OCH}_{2} \mathrm{CH}_{2} \mathrm{CH}_{2} \mathrm{CH}_{2} \mathrm{Si}\right), 18.3-18.9\left(\mathrm{SiCH}_{2} \mathrm{CH}_{2} \mathrm{CH}_{2} \mathrm{Si}\right.$ and $\begin{array}{lllllll}\left.\left(\mathrm{CH}_{3}\right) \mathrm{CH}\right), & 20.2 & \left(\mathrm{OCH}_{2} \mathrm{CH}_{2} \mathrm{CH}_{2} \mathrm{CH}_{2} \mathrm{Si}\right), \quad 22.4 & \left(\left(\mathrm{CH}_{3}\right)_{2} \mathrm{CH}\right), & 30.2 & \left(\left(\mathrm{CH}_{3}\right)_{2} \mathrm{CH}\right), & 32.4\end{array}$ $\left(\mathrm{OCH}_{2} \mathrm{CH}_{2} \mathrm{CH}_{2} \mathrm{CH}_{2} \mathrm{Si}\right), 45.0(\mathrm{CHCO}), 45.2\left(\mathrm{CH}_{2} \mathrm{Ar}\right), 64.5\left(\mathrm{OCH}_{2}\right), 127.1$ and $129.2(\mathrm{Ar}, \mathrm{C}-\mathrm{H}), 132.6$ $\left(\mathrm{SiCHCH}_{2}\right), 137.2\left(\mathrm{SiCHCH}_{2}\right), 137.8$ y $140.4\left(\mathrm{Ar}, C_{\mathrm{ipso}}\right), 174.8(C=\mathrm{O}) .{ }^{29} \mathrm{Si} \mathrm{NMR}\left(\mathrm{CDCl}_{3}\right): \delta 1.6\left(\mathrm{G}_{1}-\right.$ Si), $1.0\left(\mathrm{G}_{2}-\mathrm{Si}\right),-13.3\left(\mathrm{G}_{3}-\mathrm{SiCHCH}_{2}\right)$. Anal. Calcd. $\mathrm{C}_{58} \mathrm{H}_{106} \mathrm{O}_{2} \mathrm{Si}_{7}(1032.06 \mathrm{~g} / \mathrm{mol}): \mathrm{C}, 67.50 ; \mathrm{H}, 10.35$; Exp.: C, 67.12; H, 10.00.

\section{Macrophage differentiation and cell culture}

Blood samples were obtained from buffy coats of healthy donors from the Transfusion Center of Madrid respecting national guidelines. Peripheral blood mononuclear cells (PBMCs) were isolated by a standard Ficoll-Hypaque density gradient from buffy coats as already described. ${ }^{59}$ Monocytes were 
cultured at $0.5 \times 10^{6}$ cells $/ \mathrm{mL}$ for 7 days in RPMI medium supplemented with $10 \%$ foetal bovine serum (FBS) and $1000 \mathrm{U} / \mathrm{mL}$ of recombinant human granulocyte macrophage-colony stimulating factor (rh GM-CSF) (ImmunoTools) or $10 \mathrm{ng} / \mathrm{mL}$ of recombinant human macrophage-colony stimulating factor (rhM-CSF) (ImmunoTools) to generate M1 and M2 monocyte-derived macrophages respectively. Cytokines were added every 2 days. It was verified that the differentiated macrophages had the proper phenotype (size and morphology) for subsequent experiments (figure $\mathrm{S} 1)$.

\section{MTT assay}

Monocytes were differentiated into M1 or M2 during 7 days as mentioned above. Macrophages were treated with dendrimers or dendrons and mitochondrial metabolism was evaluated by detecting the reduction of 3-(4,5-dimethylthiazol-2-yl)-2,5-diphenyl-tetrazolium-bromide (MTT) as indicator of toxicity. Macrophages (M1 and M2) were plated (7.5x10 4 macrophages per condition, distributed in 3 wells in a 96 well plate), and treated with cationic carbosilane dendrimers or dendrons at different concentrations $(5-20 \mu \mathrm{M})$ for 24 hours. MTT was added at $0.5 \mathrm{mg} / \mathrm{mL}$ (in Dulbecco's Modified Eagle's Medium) and incubated during four hours at $37^{\circ} \mathrm{C}$. The plate was centrifuged, the supernatant removed and the reaction was stopped by adding DMSO $(50 \mu \mathrm{L}$ per well). The plate was shaked for 5 minutes and analyzed in a plate reader at $570 \mathrm{~nm}$ and the reference used was absorbance at $690 \mathrm{~nm}$. Viability was calculated by normalizing data to untreated cells, and referred to $100 \%$. Conditions which their 2xSEM (confidence interval of 95\%) do not include values equal or greater than $80 \%$ were rolled out.

\section{CASY® Cell Counter}

Viability was evaluated using the CASY ${ }^{\circledR}$ Cell Counter. Monocytes were differentiated into M1 or M2 during 7 days as mentioned above. The 7 th day $3 \times 10^{5}$ macrophages per condition (M1 or M2) were counted and turn them to the plate to adhere over night. Cationic carbosilane dendrimers or dendrons were added in the culture plate at $3 \mu \mathrm{M}$ or $5 \mu \mathrm{M}$. After 24 hours cells were washed with PBS, trypsinized, and resuspended in PBS. Samples were analyzed with the cell counter. Counts were 
corrected by the dilution factor and cells per $\mathrm{mL}$ were calculated. Viability was calculated by normalizing data to untreated cells, and referred to $100 \%$. Conditions that their 2xSEM (confidence interval of 95) does not include values equal or greater than $80 \%$ were discard.

\section{Quantitative real-time PCR}

Three combinations of different conditions were tested. The first condition was: M1 and M2 were treated with LPS $(10 \mathrm{ng} / \mathrm{ml})$, ibuprofen, $\mathrm{G}_{2} \mathrm{O}_{3}\left(\mathrm{NMe}_{3}{ }^{+}\right)_{12} \quad(\mathbf{1 8}), \mathrm{G}_{2} \mathrm{O}_{3}(\mathrm{OCOIbu})\left(\mathrm{NMe}_{3}{ }^{+}\right)_{11}$ $\mathrm{HOC}_{2} \mathrm{H}_{4} \mathrm{OG}_{3}\left(\mathrm{NMe}_{3}{ }^{+}\right)_{8}(\mathbf{1 9})$, or $\mathrm{IbuCO}_{2} \mathrm{G}_{3}\left(\mathrm{NMe}_{3}{ }^{+}\right)_{8}(\mathbf{1 7})$ at $5 \mu \mathrm{M}$ for 24 hours. The second condition was: M1 and M2 were treated with ibuprofen or compounds at $5 \mu \mathrm{M}$ for 30 minutes and then treated with LPS (10ng/ml) for 24 hours. The third condition was: M1 and M2 were treated with LPS at $10 \mathrm{ng} / \mathrm{ml}$ for 30 minutes and then were treated with ibuprofen or compounds for 24 hours. Subsequently, RNA of all samples was extracted by using the RNeasy Plus mini kit (Qiagen). RNA integrity was analyzed with Agilent 2100 bioanalyzer (Agilent Technologies) by using RNA Nano chips (Agilent Technologies). A reverse transcriptase was used to generate complementary DNA (cDNA) from RNA templates (reverse transcription) with the GoScript Reverse Transcription System (Promega). mRNA expression was analyzed by qRT-PCR using specific probe and primers for $C O X-2$ (designed by using the Universal Human Probe Roche library system, Roche Diagnostics). Results were processed with the iQ5 2.0 software (Bio-Rad). Data was normalized according to the expression levels of a housekeeping gene (TATA-binding protein, TBP), and expressed relative to the mRNA of untreated samples (relative mRNA).

\section{Cytokines release}

Macrophages (M1 and M2) were treated with LPS (Sigma-Aldrich) at 10ng/ml for 24 hours, or pre-treated with ibuprofen or compounds at $5 \mu \mathrm{M}$ for 30 minutes and after treated with LPS for 24 hours; or pre-treated with LPS for 30 minutes and after treated with ibuprofen or compounds for 24 hours. Then, supernatants were collected and amount of cytokines and chemokines were determined (TNF $\alpha$, IL-8, IL-6, CCL3 and IL-1 $\beta$ in M1 cultures; and TNF $\alpha$, IL-8, CCL3, IL-10, CXCL9and CCL2in M2 cultures) using the Flow Cytomix system (eBioscience) following the instructions given 
by manufacturer. Gallios Beckmann Coulter cytometer was used and data was analyzed by FlowCytomixPro software, version 3.0.

\section{RESULTS AND DISCUSSION}

As commented above, we report the synthesis of ibuprofen conjugated cationic carbosilane dentritic molecules through a labile ester bond. The ammonium groups decorating dendritic molecules are of the type $-\mathrm{NMe}_{3}{ }^{+}$, since carbosilane dendrimers and dendrons with more acidic $-\mathrm{NR}_{2} \mathrm{H}^{+}$ functions become insoluble if the proton is released from their structure, due to the highly hydrophobic framework of these macromolecules. To simplify the nomenclature of dendritic structures they will be named as $\mathrm{G}_{\mathrm{n}} \mathrm{O}_{3} \mathrm{Y}_{\mathrm{m}}$ for dendrimers and $\mathrm{XG}_{\mathrm{n}} \mathrm{Y}_{\mathrm{m}}$ for dendrons, where $\mathrm{G}_{\mathrm{n}}$ stands for the dendritic generation and $\mathrm{Y}_{\mathrm{m}}$ for the peripheral functions and its number; $\mathrm{O}_{3}$ denotes the polyphenoxo moiety used as a core in dendrimers, and X refers to the group placed in the focal point of dendrons.

For the synthesis of a cationic dendrimer with ibuprofen bonded through an ester bond (scheme 1) we required the presence of a hydroxyl function on the periphery for further attached of ibuprofen via Steglich esterification, and amine groups, precursors of cationic ammonium groups. With this in mind, starting from the vinyl dendrimers $\mathrm{G}_{2} \mathrm{O}_{3} \mathrm{~V}_{12}$, two consecutive thiol-ene addition reactions were carried out, following the synthetic procedure previously described for homofunctionalized dendrimers. ${ }^{57}$ First, the treatment with one eq. of 2-mercaptoethanol and subsequently with excess 2(dimethylamino)ethanethiol hydrochloride, led to dendrimer $\mathrm{G}_{2} \mathrm{O}_{3}(\mathrm{OH})\left(\mathrm{NMe}_{2} \mathrm{H}^{+}\right)_{11}$ (2), which after base treatment afforded neutral derivative $\mathrm{G}_{2} \mathrm{O}_{3}(\mathrm{OH})\left(\mathrm{NMe}_{2}\right)_{11}$ (3). Once obtained dendrimer 3, addition of ibuprofen was performed in dry DMF using DMAP (4-dymethylaminopyridine) as a base and EDCI (1-ethyl-3-(3-dimethylaminopropyl)carbodiimide) as a coupling agent, leading to ibuprofen-conjugated dendrimer $\mathrm{G}_{2} \mathrm{O}_{3}(\mathrm{OCOIbu})\left(\mathrm{NMe}_{2}\right)_{11}$ (4), which after reaction with MeI allowed us to obtain the target product $\mathrm{O}_{2} \mathrm{O}_{3}(\mathrm{OCOIbu})\left(\mathrm{NMe}_{3}{ }^{+}\right)_{11}(\mathbf{5})$ as a water soluble solid in high yields (figure 1). These compounds were characterized by NMR spectroscopy and elemental analyses. 

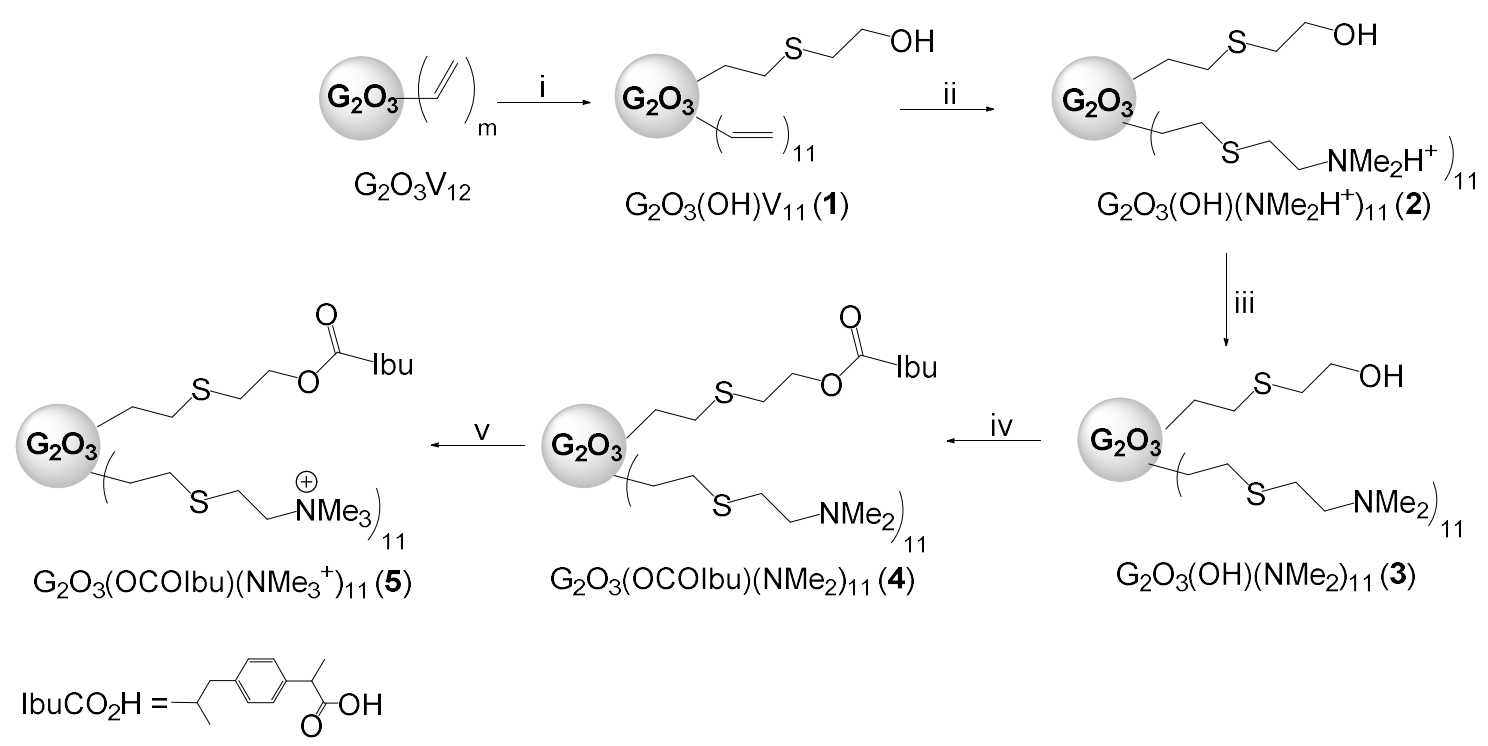

Scheme 1. Synthesis of the ibuprofen conjugated cationic dendrimer $\mathrm{G}_{2} \mathrm{O}_{3}(\mathrm{OCOIbu})\left(\mathrm{NMe}_{3}{ }^{+}\right)_{11}$ (5).i) $\mathrm{HS}\left(\mathrm{CH}_{2}\right)_{2} \mathrm{OH}, \mathrm{h} v, \mathrm{DMPA}$; ii) $\mathrm{HS}\left(\mathrm{CH}_{2}\right)_{2} \mathrm{NMe}_{2} \mathrm{HCl}$, hv, DMPA; iii) $\mathrm{NaOH}$; iv) $\mathrm{S}-(+)-$-ibuprofen, DMAP, EDCI; v) MeI.

Functionalization with first thiol compound to prepare $\mathrm{G}_{2} \mathrm{O}_{3}(\mathrm{OH}) \mathrm{V}_{11}(\mathbf{1})$ was followed by NMR, which showed the appearance of two signals at around $\delta 0.9$ and 2.5, in ${ }^{1} \mathrm{H}-\mathrm{NMR}$, and 15 and 27, in ${ }^{13} \mathrm{C}$-NMR, corresponding to the new formed chain $\mathrm{SCH}_{2} \mathrm{CH}_{2} \mathrm{Si}$. The signals due to 2-mercaptoethanol could be observed at c.a. $\delta 2.7,2.8$ and 3.7, in the ${ }^{1}$ H-NMR spectrum, and at c.a. $\delta 35$ and 60, in the ${ }^{13} \mathrm{C}-\mathrm{NMR}$ spectrum. Also, the ${ }^{1} \mathrm{H}-\mathrm{NMR}$ spectrum corroborated the presence of this moiety in the established proportion by comparison of the new signals and the ones corresponding to the nonreacted vinyl groups. The spectroscopic data of compounds $\mathbf{2}$ and $\mathbf{3}$, showed the expected signals due to the introduction of the second thiol derivative. ${ }^{57}$ For the heterofunctionalized ibuprofen-dendrimers $\mathrm{G}_{2} \mathrm{O}_{3}(\mathrm{OCOIbu})\left(\mathrm{NMe}_{2}\right)_{11}$ (4) and $\mathrm{G}_{2} \mathrm{O}_{3}(\mathrm{OCOIbu})\left(\mathrm{NMe}_{3}{ }^{+}\right)_{11}(\mathbf{5})$, confirmation of ester bond formation was done by the resonances corresponding to the methylene group directly attached to ibuprofen at c.a. $\delta 4.1$ (from $\delta 3.7$ in compound 3) and 64 in ${ }^{1} \mathrm{H}$ - and ${ }^{13} \mathrm{C}-\mathrm{NMR}$ spectra, respectively. Also, the resonances due to the presence of the ibuprofen fragment were observed (figure S3). Finally, a DOSY NMR experiment showed a unique diffusion coefficient for compounds $\mathbf{4}$ and $\mathbf{5}$. The same experiment for a mixture of the corresponding homofunctionalized dendrimer and ibuprofen showed one diffusion coefficient for each compound (figure S3). 

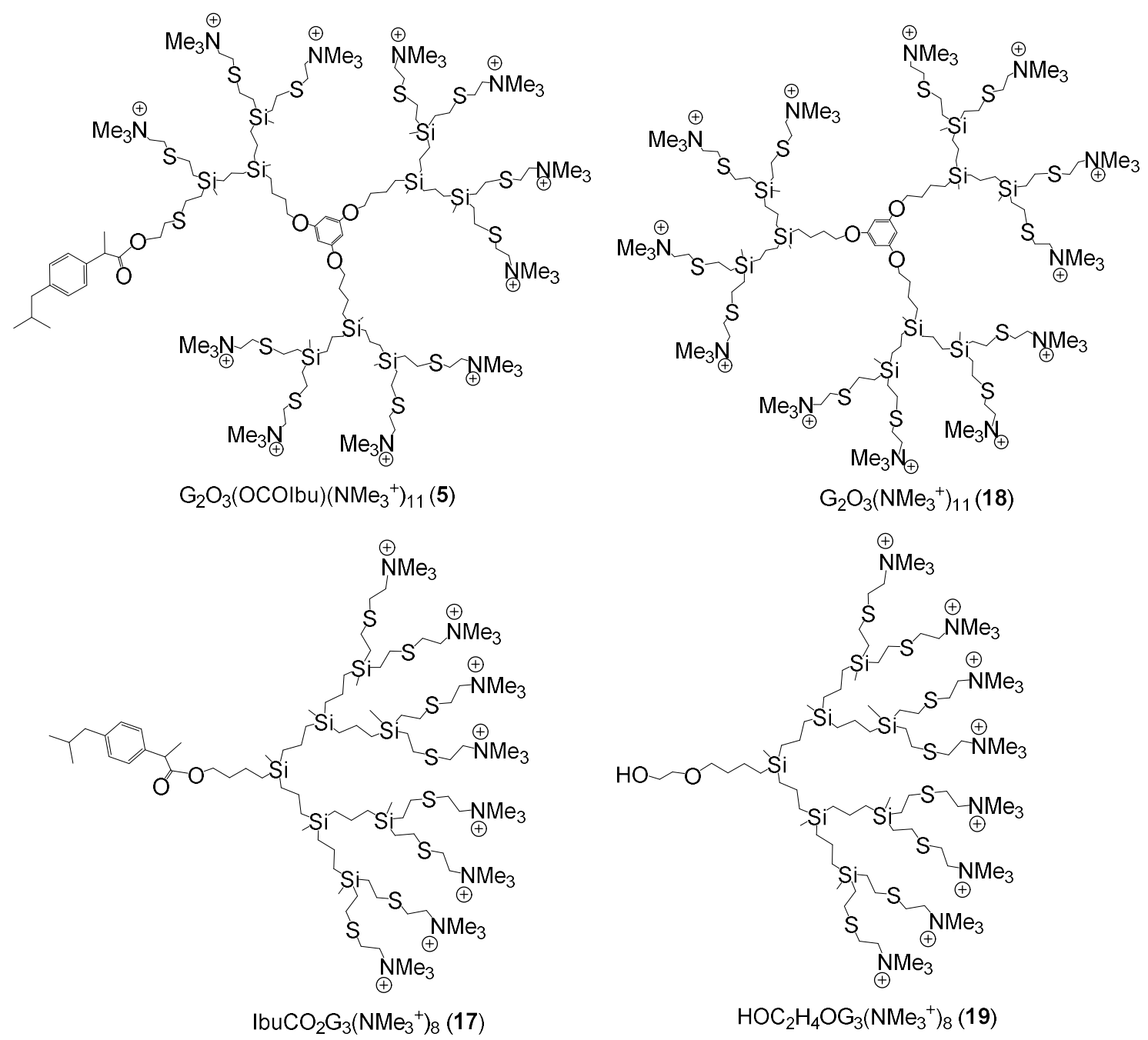

Figure 1. Drawing of selected cationic ibuprofen-conjugated and non-ibuprofen dendritic molecules. Iodide anions are omitted for clarity.

Regarding dendrons, introduction of the ibuprofen moiety at the focal point was afforded by nucleophilic substitution of the bromide atom in $\operatorname{BrG}_{\mathrm{n}} \mathrm{V}_{\mathrm{m}}(\mathrm{n}=1, \mathrm{~m}=2 ; \mathrm{n}=2, \mathrm{~m}=4 ; \mathrm{n}=3, \mathrm{~m}=8)^{58}$ by the carboxylic moiety in ibuprofen (scheme S1), avoiding the use of coupling agents. The new dendrons $\operatorname{IbuCO}_{2} \mathrm{G}_{\mathrm{n}} \mathrm{V}_{\mathrm{m}}(\mathrm{n}=1, \mathrm{~m}=2(\mathbf{6}) ; \mathrm{n}=2, \mathrm{~m}=4(7) ; \mathrm{n}=3, \mathrm{~m}=8(\mathbf{8}))$ thus formed were easily purified by simple water/Et ${ }_{2} \mathrm{O}$ extraction. The presence of this ibuprofen moiety in dendrons 6-8 was confirmed by NMR spectroscopy. The original resonances of the methylene $\mathrm{BrCH}_{2}$ disappear $(\delta 3.40$ in the ${ }^{1} \mathrm{H}-\mathrm{NMR}$ spectra) and new resonances belonging to the methylene $\mathrm{CO}_{2} \mathrm{CH}_{2}$ at the focal point bound to ibuprofen were observed at c.a. $\delta 4.0$ and c.a. $\delta 64$ in ${ }^{1} \mathrm{H}$ - and ${ }^{13} \mathrm{C}-\mathrm{NMR}$ spectra, respectively. In these spectra also the appearance of the signals due to the ibuprofen fragment could be 
observed. Next, following an identical protocol than for dendrimers (see above), the cationic dendrons $\mathrm{IbuCO}_{2} \mathrm{G}_{\mathrm{n}}\left(\mathrm{NMe}_{3}{ }^{+}\right)_{\mathrm{m}}(\mathbf{1 5 - 1 7}$, scheme S1, figure 1, figure S2) were obtained.

These compounds were characterized by NMR spectroscopy, mass spectrometry (MS) and elemental analyses. Their NMR characteristics were also analogous to those of dendrimer but for the presence of the focal chain, which resonances were as those described in dendrons 6-8. That is, the presence of the ibuprofen bound through an ester bond is confirmed by the resonances belonging to the methylene group $\mathrm{CO}_{2} \mathrm{CH}_{2}$ directly attached to this group at c.a. $\delta 4.1$ and c.a. $\delta 64$ in the ${ }^{1} \mathrm{H}$ - and ${ }^{13} \mathrm{C}-\mathrm{NMR}$ spectra, respectively. Moreover, in the MS were detected peaks corresponding to the whole cationic dendritic structure containing ibuprofen. On the other hand, HPLC did not show the presence of free ibuprofen in cationic dendrons 15-17.

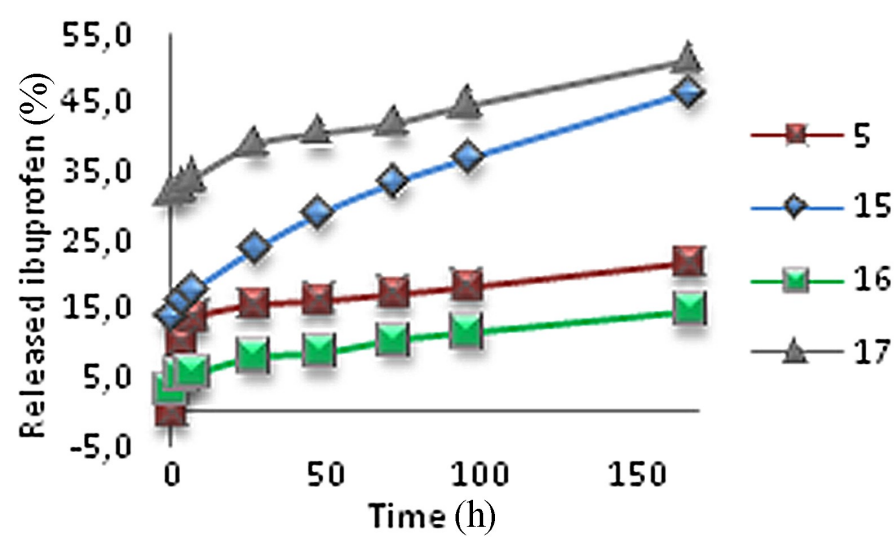

Figure 2. Release of ibuprofen (\%, HPLC) after addition of 1 unit of porcine liver esterease (PLE) per ester bond at different times (hours, h) for dendrimer $\mathrm{G}_{2} \mathrm{O}_{3}(\mathrm{OCOIbu})\left(\mathrm{NMe}_{3}{ }^{+}\right)_{11}(\mathbf{5})$ and dendrons $\mathrm{IbuCO}_{2} \mathrm{G}_{\mathrm{n}}\left(\mathrm{NMe}_{3}{ }^{+}\right)_{\mathrm{m}}(\mathrm{n}=1, \mathrm{~m}=2(\mathbf{1 5}) ; \mathrm{n}=2, \mathrm{~m}=4(\mathbf{1 6}) ; \mathrm{n}=3, \mathrm{~m}=8(\mathbf{1 7}))$.

The purpose of introducing an ibuprofen moiety in cationic derivatives $\mathbf{5}$ and $\mathbf{1 5 - 1 7}$ is obtaining a combined action between the cationic dendritic framework and the anti-inflammatory drug. Hence, the release of ibuprofen in an adequate environment should be produced. Although the synthesized compounds are stable in aqueous media, even at slightly acid $\mathrm{pH}$, it must be considered that in the organism there are hydrolytic enzymes able to break the ester bonds. For this reason, dendrimer $\mathrm{G}_{2} \mathrm{O}_{3}(\mathrm{OCOIbu})\left(\mathrm{NMe}_{3}{ }^{+}\right)_{11}$ (5) and dendrons $\mathrm{IbuCO}_{2} \mathrm{G}_{\mathrm{n}}\left(\mathrm{NMe}_{3}{ }^{+}\right)_{\mathrm{m}}$ (15-17) were incubated in the 
presence of porcine liver esterase (PLE, $1 \mu \mathrm{mol}$ ester: 1 unit) and the release of ibuprofen was monitored at several times using HPLC. After addition of esterase, a certain amount of ibuprofen, dependent on the compound, was released (figure 2). Although it turned out impossible to establish a relationship between the structure and the amount of drug released, it must be highlighted that it might be related with the conformation of these compounds in aqueous solutions. These dendritic systems contain hydrophobic, ibuprofen ligand and carbosilane framework, and hydrophilic, ammonium groups, moieties and therefore, the exposure of the hydrophobic parts in aqueous solution tend to be minimized. For this reason, the accessibility of the ester bond can randomly vary for these compounds. Addition of extra portions of esterase reactivated the released process.

Next step was to evaluate the effect of cationic systems $\mathrm{G}_{2} \mathrm{O}_{3}\left(\mathrm{NMe}_{3}{ }^{+}\right)_{12}$ (18), $\mathrm{G}_{2} \mathrm{O}_{3}(\mathrm{OCOIbu})\left(\mathrm{NMe}_{3}{ }^{+}\right)_{11}(\mathbf{5}), \mathrm{HOC}_{2} \mathrm{H}_{4} \mathrm{OG}_{3}\left(\mathrm{NMe}_{3}{ }^{+}\right)_{8}(\mathbf{1 9})$, or $\mathrm{IbuCO}_{2} \mathrm{G}_{3}\left(\mathrm{NMe}_{3}{ }^{+}\right)_{8}(\mathbf{1 7})$, bound or not with ibuprofen, on the inflammatory response mediated by macrophages. The selection of the second generation dendrimers and a third generation dendrons for these assays was done on the basis of previous studies on cationic carbosilane dendrimers for gene therapy, which showed that the best relationship between activity and toxicity were for these systems than for lower or higher generation derivatives. $^{32,47}$

The mRNA of the inducible cyclooxigenase (COX-2) expression was quantified on M1 and M2 macrophages treated with different molecules at $5 \mu \mathrm{M}$ as non-toxic doses (establish by an MTT assay and confirmed with the electronic cell sizer CASY® Cell Counter, supplementary figure S5). Separately, macrophages were treated with bacterial lipopolysaccharide (LPS), a complex glycolipid present on the outer membrane of Gram-negative bacteria, which is one of the most potent microbial initiators of inflammation. ${ }^{60}$ For those properties, LPS was used as an inflammatory activator stimulus responsible for the production of prostaglandins and inflammatory processes. Results show that none of the cationic dendritic molecules evaluated induced significantly $C O X-2 \mathrm{mRNA}$ expression (figure 3). 

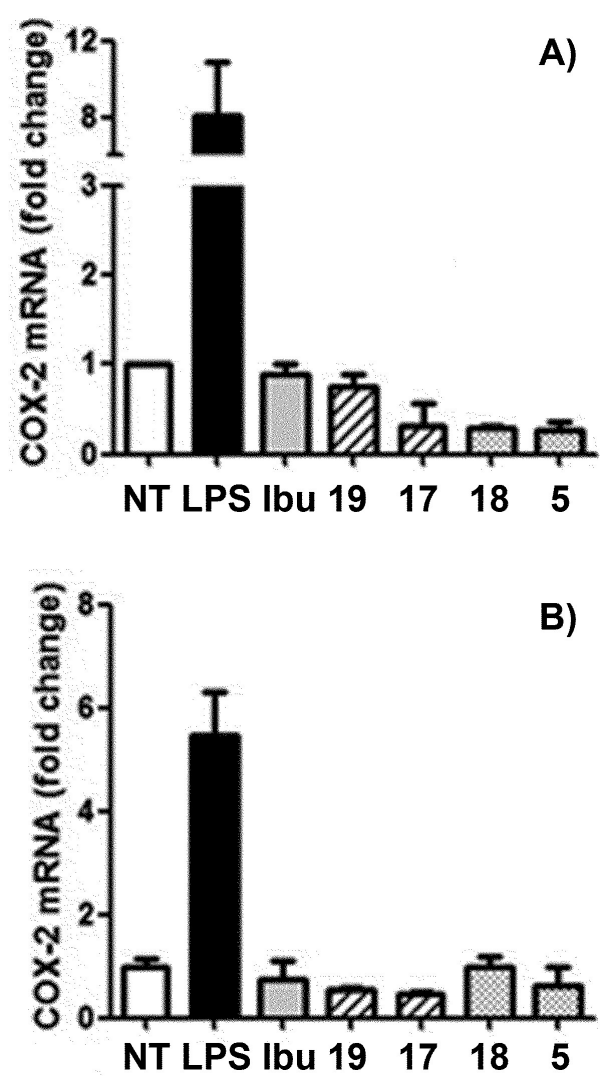

Figure 3. Quantification of gene expression on treated macrophages by Q-PCR.COX-2 mRNA expression was determined by Q-PCR in M1 (A) and M2 (B) untreated or treated with either lipopolysaccharide (LPS),ibuprofen (Ibu), $\mathrm{G}_{2} \mathrm{O}_{3}\left(\mathrm{NMe}_{3}{ }^{+}\right)_{12} \quad$ (18), $\mathrm{G}_{2} \mathrm{O}_{3}(\mathrm{OCOIbu})\left(\mathrm{NMe}_{3}{ }^{+}\right)_{11}$ (5), $\mathrm{HOC}_{2} \mathrm{H}_{4} \mathrm{OG}_{3}\left(\mathrm{NMe}_{3}{ }^{+}\right)_{8}(19)$, or $\mathrm{IbuCO}_{2} \mathrm{G}_{3}\left(\mathrm{NMe}_{3}{ }^{+}\right)_{8}$ (17) for 24 hours. Data shows relative mRNA levels; analyzed gene was normalized to TATA box binding protein expression (TBP) and referred to non-treated cells (NT). Mean and SD of triplicates are shown. One-sample t test, confidence intervals $95 \%$ of the mean (versus NT). $* p \leq 0.05$.

A lipopolysaccharide (LPS) was used as an activator stimulus over M1 and M2cells (figure 4). Macrophages were treated with dendritic derivatives previously (pre-treatment; figure 4A and 4B) or subsequently (post-treatment; figure 4C and 4D) at LPS stimulation. Also, to evaluate the effect of ibuprofen released bydendrimer5 and dendron 17, macrophages were treated with with free ibuprofen and corresponding dendrimer18 and dendron 19, simulating the in vitro release of the drug. Results show that cells treated with ibuprofen, before or after the LPS stimulus, decrease the LPS-induced expression of $C O X-2$ mRNA, both on M1 (figure 4A and 4C) and on M2 (figure 4B and 4D), as 
expected. For pre-treated M1 cultures, all tested treatments reduced the LPS-induced expression of $C O X-2$ in a similar way than ibuprofen did (figure 4A). However, post-treated M1 cells with cationic systems show improved reductions on $C O X-2$ expression compared with ibuprofen (figure 4C).Regarding M2-treated cells, and despite slight differences between pre-treatment and posttreatment, both cationic dendrons 17 and 19 and cationic dendrimer without ibuprofen 18 reduce the LPS-induced $C O X-2$ expression (figure 4B and 4D). On the contrary, ibuprofen-conjugated dendrimer 5 do not decrease the induction on $C O X-2$ expression induced by LPS over M2 (figure 4B and 4D) despite it showed great effect over M1 cells (figure 4A and 4C). Our data validate compounds $\mathrm{G}_{2} \mathrm{O}_{3}\left(\mathrm{NMe}_{3}{ }^{+}\right)_{12} \quad$ (18), $\quad \mathrm{G}_{2} \mathrm{O}_{3}(\mathrm{OCOIbu})\left(\mathrm{NMe}_{3}{ }^{+}\right)_{11} \quad$ (5), $\quad \mathrm{HOC}_{2} \mathrm{H}_{4} \mathrm{OG}_{3}\left(\mathrm{NMe}_{3}{ }^{+}\right)_{8} \quad$ (19) $\quad$ and $\mathrm{IbuCO}_{2} \mathrm{G}_{3}\left(\mathrm{NMe}_{3}{ }^{+}\right)_{8}(\mathbf{1 7})$ as non-inflammatory molecules, and at least three of them, 17, 19 and 18, reduces the LPS-induced $C O X-2$ expression in both polarization states of macrophages. These are encouraging results since COX-2 inhibition decreases the synthesis of prostaglandins, molecules involved in mediating inflammation. ${ }^{61}$
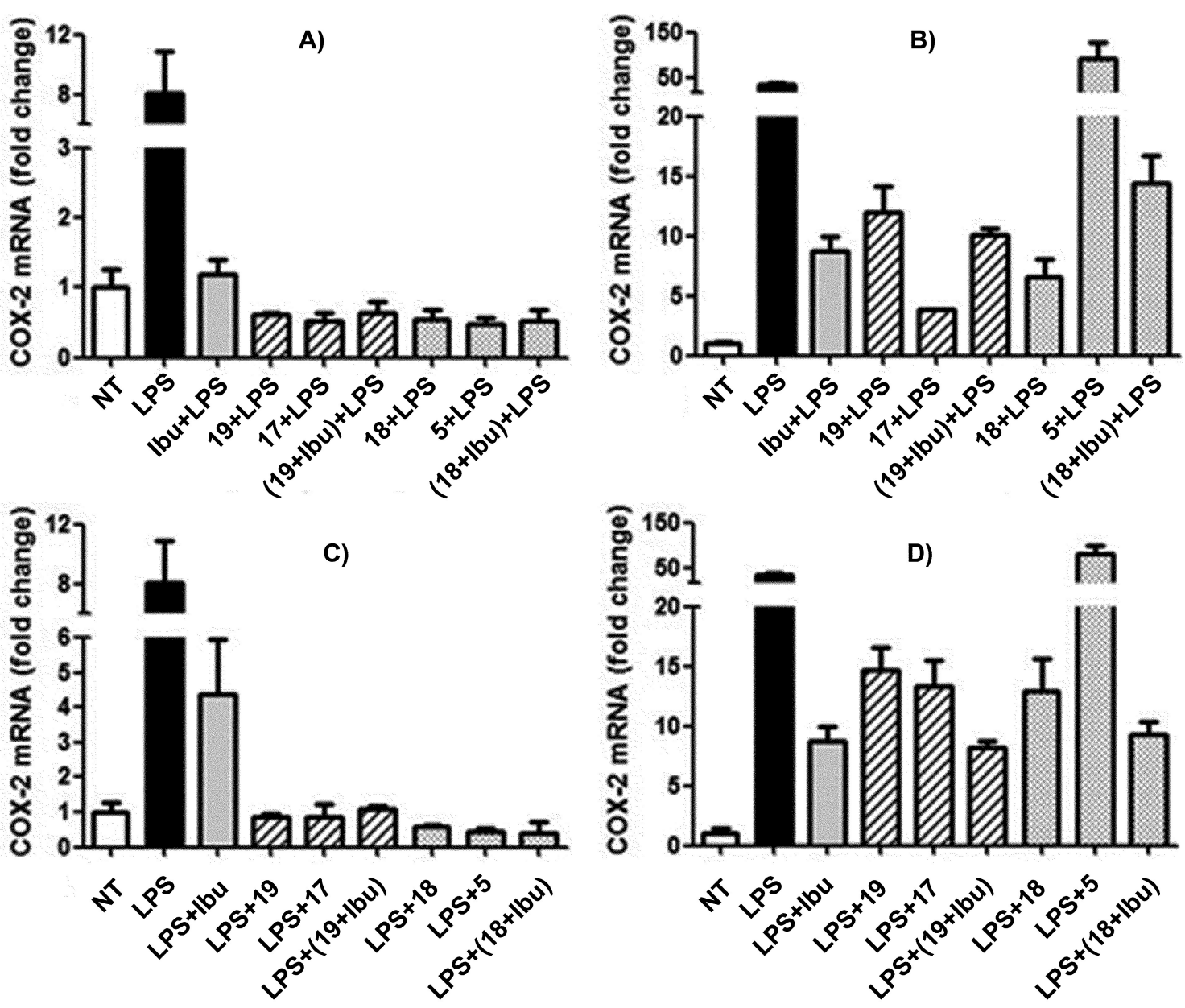
Figure 4. Quantification of gene expression on treated macrophages by Q-PCR.COX-2 mRNA expression was determined by Q-PCR in M1 (A, C) and M2 (B, D). Results on non-treated cells (NT), stimulated with lipopolysaccharide (LPS) or treated with $\mathrm{G}_{2} \mathrm{O}_{3}(\mathrm{OCOIbu})\left(\mathrm{NMe}_{3}{ }^{+}\right)_{11}$ (5) or $\mathrm{IbuCO}_{2} \mathrm{G}_{3}\left(\mathrm{NMe}_{3}{ }^{+}\right)_{8}(\mathbf{1 7})$ previously (A, B) or subsequently $(\mathrm{C}, \mathrm{D})$ at stimulating with LPS are shown. Separately, macrophages were treated with free ibuprofen and $\mathrm{G}_{2} \mathrm{O}_{3}\left(\mathrm{NMe}_{3}{ }^{+}\right)_{12}$ orHOC ${ }_{2} \mathrm{H}_{4} \mathrm{OG}_{3}\left(\mathrm{NMe}_{3}{ }^{+}\right)_{8}($ (19) previously $(\mathrm{A}, \mathrm{B})$ or subsequently $(\mathrm{C}, \mathrm{D})$ at stimulating with LPS. Data shows relative mRNA levels; analyzed gene was normalized to TATA box binding protein expression (TBP) and referred to non-treated cells (NT). Mean and SD of triplicates are shown. One-sample t test, confidence intervals $95 \%$ of the mean (versus LPS). $* \mathrm{p} \leq 0.05, * * \mathrm{p}<0.01, * * * \mathrm{p}<0.001$.

Concerning inflammatory response, when macrophages are confronted with pathogens, as in case of a bacterial infection, they secrete cytokines and chemokines; these are soluble peptides that play an important role on the development and maintenance of inflammatory process. These molecules have an effect over many signal transduction pathways that regulate cellular differentiation, and further, they are responsible of the recruitment of neutrophiles, macrophages, among other cells to specific inflammatory sites. Regarding the study of molecules involved in inflammatory processes, it is important to evaluate the molecule-induction of the inflammatory response triggered by M1but also to evaluate whether compounds allow the normal resolution of this status. Once the inflammatory response eliminates the pathogen, the anti-inflammatory macrophages (M2) play an important role down-regulating the inflammatory mediators to re-establish a homeostatic environment; this is the resolution phase of an inflammatory response. Therefore, depending on the cellular polarization state, macrophages present different cytokines and chemokinesprofiles. ${ }^{62,}{ }^{63}$ It is well known that several cytokines are involved in particle-induced inflammation, ${ }^{64}$ hence, the evaluation of cationic dendritic systems with and without bound ibuprofen over cytokines release by LPS-stimulated M1 macrophages is interesting. Also, the effect of dendrons and dendrimers over M2 cells should be 
evaluated to determine whether these treatments could improve the effect of ibuprofen, either downloading proinflammatory or raising anti-inflammatory cytokines.

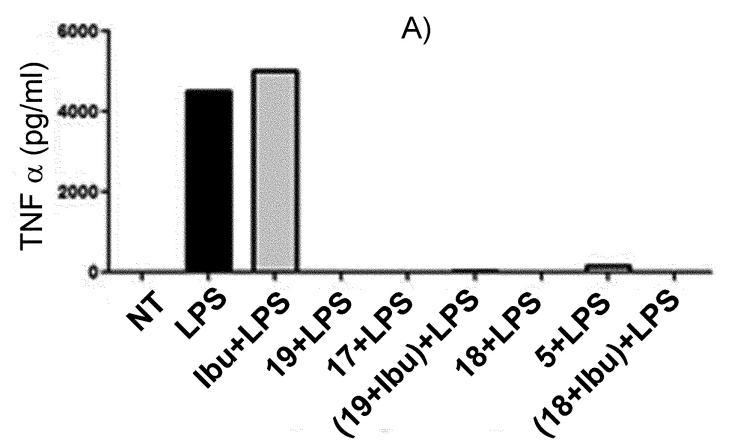

C)
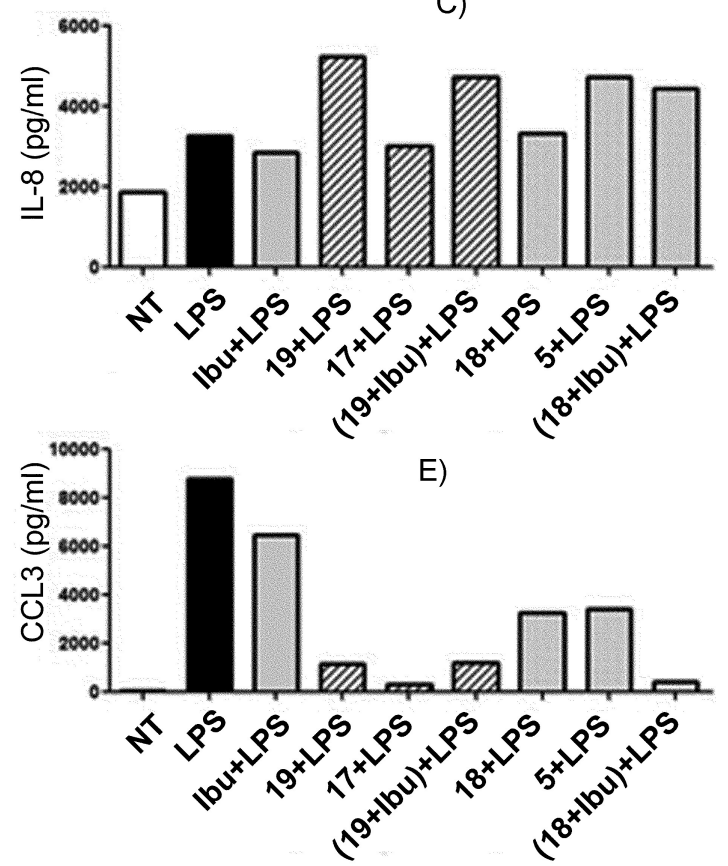

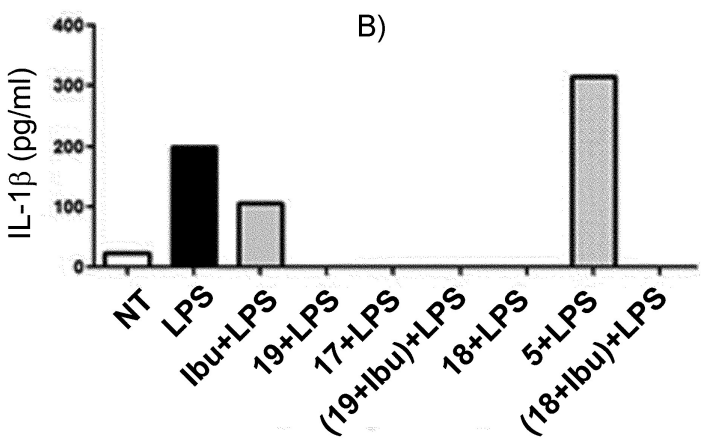

D)

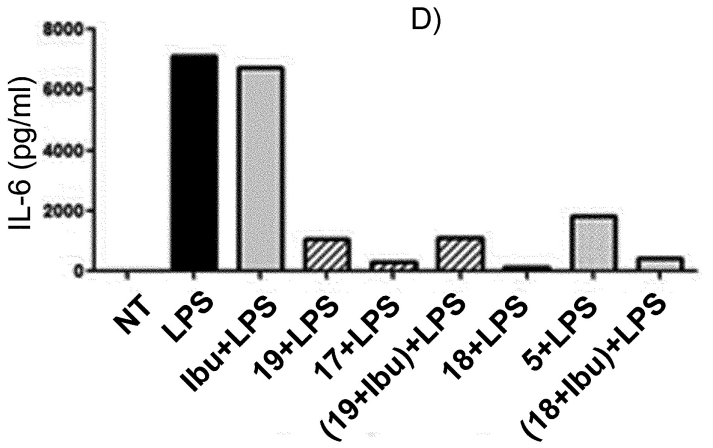

Figure 5. Cytokines release by M1 macrophages. Quantification of TNF $\alpha$ (A), IL-1 $\beta$ (B), IL-8 (C), IL-6 (D) and CCL3 (E) release in M1 cultures supernatants. Shown non-treated cells (NT), stimulated with LPS (LPS), and pre-treated with ibuprofen (Ibu+LPS), $\mathrm{HOC}_{2} \mathrm{H}_{4} \mathrm{OG}_{3}\left(\mathrm{NMe}_{3}{ }^{+}\right)_{8}$ (19+LPS), $\mathrm{IbuCO}_{2} \mathrm{G}_{3}\left(\mathrm{NMe}_{3}^{+}\right)_{8}(\mathbf{1 7}+\mathrm{LPS}), \mathrm{G}_{2} \mathrm{O}_{3}\left(\mathrm{NMe}_{3}{ }^{+}\right)_{12}$ (18+LPS) or $\mathrm{G}_{2} \mathrm{O}_{3}(\mathrm{OCOIbu})\left(\mathrm{NMe}_{3}^{+}\right)_{11}$ (5+LPS) for 30 min previously at stimulating with LPS for $24 \mathrm{~h}$. Also shown macrophages treated with $\mathrm{HOC}_{2} \mathrm{H}_{4} \mathrm{OG}_{3}\left(\mathrm{NMe}_{3}{ }^{+}\right)_{8}((\mathbf{1 9}+\mathrm{Ibu})+\mathrm{LPS})$ or $\mathrm{G}_{2} \mathrm{O}_{3}\left(\mathrm{NMe}_{3}{ }^{+}\right)_{12}((\mathbf{1 8}+\mathrm{Ibu})+\mathrm{LPS})$ and free ibuprofen for 30 min previously at stimulating with LPS for $24 \mathrm{~h}$. Individual values from one donor are shown.

For these assays, macrophages were treated with ibuprofen-conjugated dendrimer (5) and dendron (17), both previously (pre-treatment; figure 5 and figure S6) or subsequently (post-treatment; figure 6 
and figure S7) at LPS stimulation. Macrophages were also treated with ibuprofen in the presence of cationic dendrimer18 and dendron 19. As expected and in line with previous results, ${ }^{63}$ LPS activates M1 macrophages to produce pro-inflammatory cytokines such as tumor necrosis factor- $\alpha$ (TNF$\alpha$ )(figure 5A and 6A), IL-1 $\beta$ (figure 5B and 6B), also slightly increases the production of IL-8 (figure 5C and 6C) and in a higher way affects the IL-6 (figure 5D and 6D) and CCL3 (figure 5E and 6E) release. ${ }^{62}$ On the other hand, LPS activates M2 macrophages to produce anti-inflammatory cytokines such as IL-10 (figure S6 and S7), confirming that these cells are prepared to respond to stimuli releasing anti-inflammatory cytokines. ${ }^{42,} 65$ Ibuprofen-treated macrophages do not modify some cytokines release, since not all of the evaluated cytokines are related with the ibuprofen pathway (figure S6 and S7). Concerning proinflammatory cytokines, dendrons(19 and 17) or dendrimers (18 and 5) treatment blocks the TNF- $\alpha$ release by M1 cells (figure 5A and 6A), showing an important advantage compared with ibuprofen treatment, since it does not affect the TNF- $\alpha$ production. TNF- $\alpha$ promotes COX-2 induction and prostaglandin $\mathrm{E}_{2}\left(\mathrm{PGE}_{2}\right)$ production, ${ }^{66}$ which is the enzymatic product of COX-2. ${ }^{67}$ Given that compounds 19, 17 and $\mathbf{1 8}$ blocks the expression of COX-2 induced by LPS, would be interesting to study whether they have a direct effect on this molecule or whether they are acting upstream in the cell signaling pathway. In this regard, COX-2 mRNA could be induced by IL$1 \beta$ and IL-8, and interestingly, ${ }^{68-72}$ dendrons (19 and 17) and dendrimer $\mathbf{1 8}$ treatment reduces the IL$1 \beta$ production by M1 macrophages, both pretreatment (figure 5B) and post-treatment (figure 6B), whilst combination of ibuprofen-conjugated cationic dendrimer 5 with LPS increases the IL-1 $\beta$ release compared with LPS activation (figure 5B and 6B). It has been reported that compounds affecting TNF- $\alpha$ and COX-2 expression, also affect the IL- 6 and IL- 8 expression, ${ }^{70,73}$ therefore, the evaluation of these cytokines is interesting. The data show that $\mathbf{1 7}$ and $\mathbf{1 8}$ pre-treatment were not affecting the IL-8 release compared with LPS treatment (figure 5C and S6C), suggesting an ibuprofen-like profile over M1 and M2 cells, while 19 pre-treatment slightly increase the IL-8 release by M1 regardless it was combine or not with free ibuprofen (figure 5C). Moreover, dendrons $(\mathbf{1 9}, \mathbf{1 7})$ or dendrimers $(\mathbf{1 8}, \mathbf{5})$ treatments decrease the IL-6 (figure 5D and 6D) which is usually produced by 
viral infection, and also reduce CCL3 release by proinflammatory macrophages (figure 5E and 6E), despite they were activated with LPS (previously or subsequently, figure 5 and 6, respectively). Ibuprofen-conjugated dendrimer5 was the only one of all tested derivatives that increased TNF $\alpha$ (figure S6A and S7A) and CCL3 (figure S6E and S7E) secretion by M2 when combined with LPS, indicating that M2-treated cells acquire inflammatory properties. Given these results together with the induction of $C O X-2$ expression (figure 4B) and changes in the cytokine profile of the M1 (figure 5 and 6), dendrimer 5 was discard as anti-inflammatory treatment, despite induce a higher secretion of IL-10 than LPS (figure S6B and S7B).
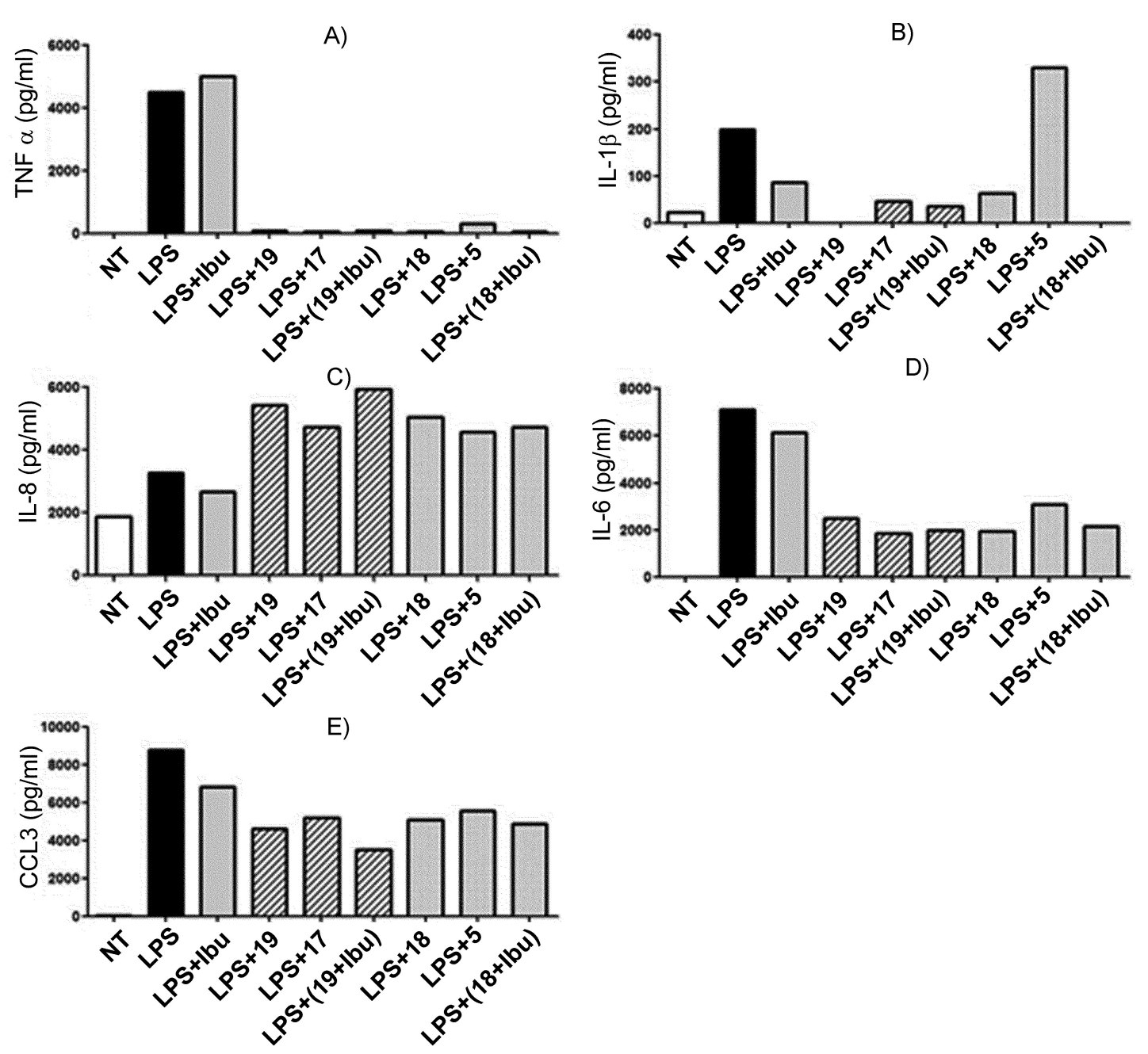

Figure 6. Cytokines release by M1 macrophages. Quantification of TNF $\alpha$ (A), IL-1 $\beta$ (B), IL-8 (C), IL-6 (D) and CCL3 (E) release in M1 cultures supernatants. Shown non-treated cells (NT), stimulated with LPS (LPS), and treated with LPS for 30 minutes and then with ibuprofen (LPS+Ibu), $\mathrm{HOC}_{2} \mathrm{H}_{4} \mathrm{OG}_{3}\left(\mathrm{NMe}_{3}{ }^{+}\right)_{8} \quad(\mathrm{LPS}+19), \quad \mathrm{IbuCO}_{2} \mathrm{G}_{3}\left(\mathrm{NMe}_{3}{ }^{+}\right)_{8} \quad(\mathrm{LPS}+\mathbf{1 7}), \mathrm{G}_{2} \mathrm{O}_{3}\left(\mathrm{NMe}_{3}{ }^{+}\right)_{12} \quad(\mathrm{LPS}+\mathbf{1 8})$ or 
$\mathrm{G}_{2} \mathrm{O}_{3}(\mathrm{OCOIbu})\left(\mathrm{NMe}_{3}{ }^{+}\right)_{11}$ (LPS+5)for 24 hours. Also shown macrophages treated with LPS for 30 minutes and after treated with free ibuprofen and $\mathrm{HOC}_{2} \mathrm{H}_{4} \mathrm{OG}_{3}\left(\mathrm{NMe}_{3}{ }^{+}\right)_{8}((\mathrm{LPS}+(\mathbf{1 9}+\mathrm{Ibu}))$ or $\mathrm{G}_{2} \mathrm{O}_{3}\left(\mathrm{NMe}_{3}{ }^{+}\right)_{12}((\mathrm{LPS}+(\mathbf{1 8}+\mathrm{Ibu}))$ for 24 hours. Individual values from one donor are shown.

Summing up, cationic ibuprofen-conjugated dendron 17 presents the best results as antiinflammatory agent. It reduces the LPS-induced $C O X-2$ expression and shows an improved effect compared with free ibuprofen used as control, since it decreases the inflammatory cytokines in a higher way that ibuprofen do it. Dendron 17 decreases TNF $\alpha$ release (figure 5A, 6A, S6A and S7A), cytokine involved in systemic inflammation that activates neuthophils, IL-1 $\beta$ (figure 5B and 6B), IL-6 (figure 5D and 6D) and CCL3 (figure 5E, 6E, S6E and $\mathrm{S7E}$ ), that is a cytokine involved in the recruitment and activation of leukocytes in the acute inflammatory state. Further and interestingly, post-treatment with dendron 17 decreases the expression of CCL2 by M2. CCL2 regulates the migration and infiltration of monocytes, memory $\mathrm{T}$ lymphocytes, and natural killer (NK) cells at inflammatory sites. \{Deshmane, 2009 \#525\} Besides, dendron 17 do not increase the expression of IL8 (figure 5C, 6C, S6C and S7C) nor CXCL9 (figure S7C and S7D) by M2, indicating that do not induce recruitment of leukocytes to infection sites. These results are very encouraging because they define the dendron $\mathrm{IbuCO}_{2} \mathrm{G}_{3}\left(\mathrm{NMe}_{3}{ }^{+}\right)_{8}(\mathbf{1 7})$ as a drug for a new anti-inflammatory therapy.

\section{CONCLUSIONS}

Cationic carbosilane dendrimers and dendrons with ibuprofen bound through an ester bond can be obtained by esterification or nucleophilic substitution, respectively, and further thiol-ene addition. This ester bond can be used to release the antiinflammatory moiety, as has been demonstrated by treating these compounds with esterease (PLE).

The inflammatory response of ibuprofen-conjugated dendritic molecules was evaluated on macrophages (M1 and M2) and the results were compared with those obtained for corresponding nonibuprofen counterparts, both in the presence or absence of free ibuprofen. These assays highlight that 
ibuprofen-conjugated dendron $\mathrm{IbuCO}_{2} \mathrm{G}_{3}\left(\mathrm{NMe}_{3}{ }^{+}\right)_{8}(17)$ reduces inflammatory responses in a better way than ibuprofen does. Taking into account the potential biomedical applications of this type of compounds, for example as microbicides or gene carriers, and that the triggering of inflammatory processes is a handicap for their applications, these results suggest that introduction of ibuprofen to cationic carbosilane dendrimers and dendrons would diminish problems associated to their use.

\section{ACKNOWLEDGMENTS}

This work has been supported by grants from CTQ-2014-54004-P (MINECO) and Consortium NANODENDMED ref S2011/BMD-2351 (CAM) to University of Alcalá (UAH). This work has been cofinanced by Instituto de Salud Carlos III (Subdirección General de Evaluación) and Fondo Europeo de Desarrollo Regional (FEDER), RETIC PT13/0010/0028, Fondo de Investigación Sanitaria (FIS) (grant number PI13/02016 and PI12/0014), Comunidad de Madrid (grant numbers S-2010/BMD2332) to HGUGM. This work has been (partially) funded by the RD12/0017/0037, as part of Acción Estratégica en Salud, Plan Nacional de Investigación Científica, Desarrollo e Innovación Tecnológica 2008-2011 (HGUGM). CIBER-BBN is an initiative funded by the VI National R\&D\&i Plan 20082011, Iniciativa Ingenio 2010, Consolider Program, CIBER Actions and financed by the Instituto de

Salud Carlos III with assistance from the European Regional Development Fund. E. F.-P. acknowledges MINECO for a fellowship. This work was supported partially by a Marie Curie International Research Staff Exchange Scheme Fellowship within the 7th European Community Framework Program, project No. PIRSES-GA-2012-316730 NANOGENE, co-financed by the Polish Ministry of Science and Higher Education (grant No. W21/7PR/ 2013) and CYTED 214RT0482 to UAH and HGUGM. We want to particularly acknowledge to the Spanish HIV HGM BioBank that is supported by the Spanish Instituto de Salud Carlos III and is integrated in the Spanish AIDS Research Network. The authors thank Dr. Laura Diaz, of the Flow Cytometry Unit (IiSGM, Madrid, Spain) and Dr. Ma Isabel Clemente, of Culture Unit (IiSGM, Madrid, Spain) for their technical assistance. 


\section{REFERENCES}

1. Elvers, K. T.; Wright, S. J. L. Antibacterial activity of the anti-inflammatory compound ibuprofen. Lett. Appl. Microbiol. 1995, 20, 82-84.

2. Núñez, C.; Fernández-Lodeiro, A.; Fernández-Lodeiro, J.; Carballo, J.; Capelo, J. L.; Lodeiro, C. Synthesis, spectroscopic studies and in vitro antibacterial activity of Ibuprofen and its derived metal complexes. Inorg. Chem. Commun. 2014, 45, 61-65.

3. Al-Janabi, A. A. H. S. In vitro antibacterial activity of Ibuprofen and acetaminophen. 2010, 2 , $105-8$.

4. Konstan, M. W.; Byard, P. J.; Hoppel, C. L.; Davis, P. B. Effect of high-dose ibuprofen in patients with cystic fibrosis. N. Engl. J. Med. 1995, 332, 848-54.

5. Seibert, K.; Masferrer, J. L. Role of inducible cyclooxygenase (COX-2) in inflammation. Receptor 1994, 4, 17-23.

6. Ricciotti, E.; Fitzgerald, G. A. Prostaglandins and inflammation. Arterioscler. Thromb. Vasc. Biol. 2011, 31, 986-1000.

7. Anderson, G. D.; Hauser, S. D.; McGarity, K. L.; Bremer, M. E.; Isakson, P. C.; Gregory, S. A. Selective inhibition of cyclooxygenase (COX)-2 reverses inflammation and expression of COX-2 and interleukin 6 in rat adjuvant arthritis. J. Clin. Invest. 1996, 97, 2672-9.

8. Milani, M.; Iacobelli, P. Vaginal use of Ibuprofen isobutanolammonium (ginenorm): efficacy, tolerability, and pharmacokinetic data: a review of available data. ISRN Obstet. Gynecol. 2012, 2012, 673131.

9. Dall, L.; Peterson, S.; Simmons, T.; Dall, A. Rapid resolution of cellulitis in patients managed with combination antibiotic and anti-inflammatory therapy. Cutis 2005, 75, 177-180.

10. Dar, U. Benzoyl-free formula acne treatment solution. CA2680502A1, 2011.

11. Hage, D. S.; Noctor, T. A. G.; Wainer, I. W. Characterization of the protein binding of chiral drugs by high-performance affinity chromatography. Interactions of R- and S-ibuprofen with human serum albumin. J. Chromatogr. A 1995, 693, 23-32.

12. Agarwal, V.; Siddiqui, A.; Ali, H.; Nazzal, S. Dissolution and powder flow characterization of solid self-emulsified drug delivery system (SEDDS). Int. J. Pharm. 2009, 366, 44-52.

13. Kutmalge, M. D.; Jadhav, A. N.; Ratnaparkhi, M. P.; Chaudhari, S. P. Sustained release drug delivery system. Pharma. Sci. Monit. 2014, 5, 191-208.

14. Chen, Y.; Li, N.; Yang, Y.; Liu, Y. A dual targeting cyclodextrin/gold nanoparticle conjugate as a scaffold for solubilization and delivery of paclitaxel. RSC Adv. 2015, 5, 8938-8941.

15. Lin, Y. H.; Lin, J. H.; Chou, S. C.; Chang, S. J.; Chung, C. C.; Chen, Y. S.; Chang, C. H. Berberine-loaded targeted nanoparticles as specific Helicobacter pylori eradication therapy: in vitro and in vivo study. Nanomedicine 2015, 10, 57-71.

16. Tanner, P.; Baumann, P.; Enea, R.; Onaca, O.; Palivan, C.; Meier, W. Polymeric Vesicles: From Drug Carriers to Nanoreactors and Artificial Organelles. Acc. Chem. Res. 2011, 44, 1039-1049.

17. Liechty, W. B.; Kryscio, D. R.; Slaughter, B. V.; Peppas, N. A. Polymers for Drug Delivery Systems. Annu. Rev. Chem. Biomol. Eng. 2010, 1, 149-173.

18. Allen, T. M.; Cullis, P. R. Drug Delivery Systems: Entering the Mainstream. Science 2004, 303, 1818-1822.

19. Cho, K.; Wang, X.; Nie, S.; Chen, Z. G.; Shin, D. M. Therapeutic Nanoparticles for Drug Delivery in Cancer. Clin. Cancer Res. 2008, 14, 1310-1316.

20. Noriega-Luna, B.; Godinez, L. A.; Rodríguez, F. J.; Rodríguez, A.; Zaldivar-Lelo de Larrea, G.; Sosa-Ferreyra, C. F.; Mercado-Curiel, R. F.; Manriquez, J.; Bustos, E. Applications of dendrimers in drug delivery agents, diagnosis, therapy and detection. J. Nanomater. 2014, 2014, ID 507273.

21. Jain, N. K.; Mishra, V.; Tare, M. S.; Tripathi, P. K. The development, characterization and in vivo anti-ovarian cancer activity of poly(propylene imine) (PPI)-antibody conjugates containing encapsulated paclitaxel. Nanomedicine: NBM 2015, 11, 207-218. 
22. He, X.; Alves, C. S.; Oliveira, N.; Rodrigues, J.; Zhu, J.; Banyai, I.; Tomas, H.; Shi, X. RGD peptide-modified multifunctional dendrimer platform for drug encapsulation and targeted inhibition of cancer cells. Colloids Surf. B 2015, 125, 82-89.

23. Huang, B.; Otis, J.; Joice, M.; Kotlyar, A.; Thomas, T. P. PSMA-Targeted Stably Linked "Dendrimer-Glutamate Urea-Methotrexate" as a Prostate Cancer Therapeutic. Biomacromolecules 2014, 15, 915-923.

24. Gillies, E. R.; Fréchet, J. M. J. Dendrimers and dendritic polymers in drug delivery. Drug Discov. Today 2005, 10, 35-43.

25. Grayson, S. M.; Fréchet, J. M. J. Convergent Dendrons and Dendrimers: from Synthesis to Applications. Chem. Rev. 2001, 101, 3819-3868.

26. Pang, J. D.; Zhuang, B. X.; Mai, K.; Chen, R. F.; Wang, J.; Zhang, L. M. Click modification of helical amylose by poly(L-lysine) dendrons for non-viral gene delivery. Mater. Sci. Eng. C 2015, 49, 485-492.

27. Kumari, M.; Gupta, S.; Achazi, K.; Boettcher, C.; Khandare, J.; Sharma, S. K.; Haag, R. Dendronized Multifunctional Amphiphilic Polymers as Efficient Nanocarriers for Biomedical Applications. Macromol. Rapid Commun. 2015, 36, 254-261.

28. Peña-González, C. E.; García-Broncano, P.; Ottaviani, M. F.; Cangiotti, M.; Fattori, A.; Hierro-Oliva, M.; González-Martín, M. L.; Pérez-Serrano, J.; Gómez, R.; Muñoz-Fernández, M. Á.; Sánchez-Nieves, J.; de la Mata, F. J. Dendronized Anionic Gold Nanoparticles: Synthesis, Characterization and Antiviral Activity. Chem. Eur. J. 2016, 22, 2987-2999.

29. Fornaguera, C.; Grijalvo, S.; Galan, M.; Fuentes-Paniagua, E.; de la Mata, F. J.; Gomez, R.; Eritja, R.; Calderó, G.; Solans, C. Novel non-viral gene delivery systems composed of carbosilane dendron functionalized nanoparticles prepared from nano-emulsions as non-viral carriers for antisense oligonucleotides. Int. J. Pharm. 2015, 478, 113-123.

30. Lamanna, G.; Russier, J.; Dumortier, H.; Bianco, A. Enhancement of anti-inflammatory drug activity by multivalent adamantane-based dendrons. Biomaterials 2012, 33, 5610-5617.

31. Kose, M. M.; Onbulak, S.; Yilmaz, I. I.; Sanyal, A. Orthogonally "Clickable" Biodegradable Dendrons. Macromolecules 2011, 44, 2707-2714.

32. Fuentes-Paniagua, E.; Serramía, M. J.; Sánchez-Nieves, J.; Álvarez, S.; Muñoz-Fernández, M. A.; Gómez, R.; de la Mata, F. J. Fluorescein Labelled Cationic Carbosilane Dendritic Systems for Biological Studies. Eur. Polym. J. 2015, 71, 61-72.

33. Galán, M.; Fuentes-Paniagua, E.; de la Mata, F. J.; Gómez, R. Heterofunctionalized carbosilane dendritic systems: bifunctionalized dendrons as building blocks versus statistically decorated dendrimers. Organometallics 2014, 33, 3977-3989.

34. Serramía, M. J.; Álvarez, S.; Fuentes-Paniagua, E.; Clemente, M. I.; Sánchez-Nieves, J.; Gómez, R.; de la Mata, J.; Muñoz-Fernández, M. A. In vivo delivery of siRNA to the brain by carbosilane dendrimer. J. Controll. Rel. 2015, 200, 60-70.

35. Kolhe, P.; Khandare, J.; Pillai, O.; Kannan, S.; Lieh-Lai, M.; Kannan, R. M. Preparation, cellular transport, and activity of polyamidoamine-based dendritic nanodevices with a high drug payload. Biomaterials 2006, 27, 660-669.

36. Kurtoglu, Y. E.; Mishra, M. K.; Kannan, S.; Kannan, R. M. Drug release characteristics of PAMAM dendrimer-drug conjugates with different linkers. Int. J. Pharm. 2010, 384, 189-194.

37. Ebru-Koc, F.; Senel, M. Solubility enhancement of Non-Steroidal Anti-Inflammatory Drugs (NSAIDs) using polypolypropylene oxide core PAMAM dendrimers. Int. J. Pharm. 2013, 451, 1822.

38. Yiyun, C.; Tongwen, X. Dendrimers as Potential Drug Carriers. Part I. Solubilization of NonSteroidal Anti-Inflammatory Drugs in the Presence of Polyamidoamine Dendrimers. Eur. J. Med. Chem. 2005, 40, 1188-1192.

39. Mantovani, A.; Sica, A.; Locati, M. New vistas on macrophage differentiation and activation. Eur. J. Immunol. 2007, 37, 14-16.

40. Benoit, M.; Desnues, B.; Mege, J. L. Macrophage polarization in bacterial infections. 2008, $181,3733-9$. 
41. Gordon, S.; Taylor, P. R. Monocyte and macrophage heterogeneity. Nat. Rev. Immunol. 2005, 5, 953-964.

42. Mantovani, A.; Sica, A.; Sozzani, S.; Allavena, P.; Vecchi, A.; Locati, M. The chemokine system in diverse forms of macrophage activation and polarization. Trends Immunol. 2004, 25, 677686.

43. Fleetwood, A. J.; Lawrence, T.; Hamilton, J. A.; Cook, A. D. Granulocyte-macrophage colony-stimulating factor (CSF) and macrophage CSF-dependent macrophage phenotypes display differences in cytokine profiles and transcription factor activities: implications for CSF blockade in inflammation. J. Immunol. 2007, 178, 5245-5252.

44. Gordon, S.; Martinez, F. O. Alternative activation of macrophages: mechanism and functions. Immunity 2010, 32, 593-604.

45. Doncel, G. F.; Chandra, N.; Fichorova, R. N. Preclinical assessment of the proinflammatory potential of microbicide candidates. J. Acquir. Immune Defic. Syndr. 2004, 37, Suppl 3, S174-180.

46. Fichorova, R. N.; Tucker, L. D.; Anderson, D. J. The molecular basis of nonoxynol-9-induced vaginal inflammation and its possible relevance to human immunodeficiency virus type 1 transmission. J. Infect. Dis. 2001, 184, 418-428.

47. Bermejo, J. F.; Ortega, P.; Chonco, L.; Eritja, R.; Samaniego, R.; Mullner, M.; de Jesús, E.; de la Mata, F. J.; Flores, J. C.; Gómez, R.; Muñoz-Fernández, A. Water-soluble carbosilane dendrimers: Synthesis biocompatibility and complexation with oligonucleotides; Evaluation for medical applications. Chem. Eur. J. 2007, 13, 483-495.

48. Sánchez-Nieves, J.; Perisé-Barrios, A. J.; Ortega, P.; Corbí, Á. L.; Domínguez-Soto, Á.; Muñoz-Fernández, M. Á.; Gómez, R.; de la Mata, F. J. Study of cationic carbosilane dendrimers as potential activating stimuli in macrophages. RSC Adv. 2013, 3, 23445-23453.

49. Pedziwiatr-Werbicka, E.; Fuentes, E.; Dzmitruk, V.; Sánchez-Nieves, J.; Sudas, M.; Drozd, E.; Shakhbazau, A.; Shcharbin, D.; de la Mata, F. J.; Gómez-Ramirez, R.; Muñoz-Fernández, M. A.; Bryszewska, M. Novel 'SiC' carbosilane dendrimers as carriers for anti-HIV nucleicacids: Studies on complexation and interaction with blood cells. Colloids Surf B Biointerfaces 2013, 109, 183- 189.

50. Heredero-Bermejo, I.; Sánchez-Nieves, J.; Copa-Patiño, J. L.; Soliveri, J.; Gómez, R.; de la Mata, F. J.; Pérez-Serrano, J. In vitro anti-Acanthamoeba polyphaga synergistic effect of chlorhexidine and cationic carbosilane dendrimers against both trophozoites and cysts forms. Int. J. Pharm. 2016, 509, 1-7.

51. Fuentes-Paniagua, E.; Sánchez-Nieves, J.; Fernández-Ezequiel, A.; Hernández-Ros, J. M.; Soliveri, J.; Copa-Patiño, J. L.; Gómez, R.; de la Mata, F. J. Structure-Activity Relationship Study of Cationic Carbosilane Dendritic Systems as Antibacterial Agents. RSC Adv. 2016, 6, 7022-7033.

52. Perisé-Barrios, A. J.; Jiménez, J. L.; Domínguez-Soto, Á.; de la Mata, F. J.; Corbí, A. L.; Gómez, R.; Muñoz-Fernández, M. Á. Carbosilane dendrimers as gene delivery agents for the treatment of HIV infection. J. Controll. Release 2014, 184, 51-57.

53. Neibert, K.; Gosein, V.; Sharma, A.; Khan, M.; Whitehead, M. A.; Maysinger, D.; Kakkar, A. "Click" Dendrimers as Anti-inflammatory Agents: With Insights into Their Binding from Molecular Modeling Studies. Mol. Pharmaceutics 2013, 10, 2502-2508.

54. Chauhan, A. S. D., P. V.; Jain, N. K.; Tomalia, D. A. Unexpected in vivo anti-inflammatory activity observed for simple, surface functionalized poly(amidoamine) dendrimers. Biomacromolecules 2009, 10, 1195-1202.

55. Qandil, A. M. Prodrugs of Nonsteroidal Anti-Inflammatory Drugs (NSAIDs), More Than Meets the Eye: A Critical Review. Int. J. Mol. Sci. 2012, 13, 17244-17274.

56. Goonewardena, S. N.; Kratz, J. D.; Zong, H.; Desai, A. M.; Tang, S.; Emery, S.; Baker, J. R.; Huang, B. Design considerations for PAMAM dendrimer therapeutics. Bioorg. Med. Chem. Lett. 2013, 23, 2872-2875.

57. Fuentes-Paniagua, E.; Hernández-Ros, J. M.; Sánchez-Milla, M.; Camero, M. A.; Maly, M.; Pérez-Serrano, J.; Copa-Patiño, J. L.; Sánchez-Nieves, J.; Soliveri, J.; Gómez, R.; Javier de la Mata, F. Carbosilane cationic dendrimers synthesized by thiol-ene click chemistry and their use as antibacterial agents. $R S C A d v$. 2014, 4, 1256-1265. 
58. Fuentes-Paniagua, E.; Peña-González, C. E.; Galán, M.; Gómez, R.; de la Mata, F. J.; SánchezNieves, J. Thiol-Ene Synthesis of Cationic Carbosilane Dendrons: a New Family of Synthons. Organometallics 2013, 32, 1789-1796.

59. Garcia-Merino, I.; de Las Cuevas, N.; Jimenez, J. L.; Gallego, J.; Gomez, C.; Prieto, C.; Serramia, M. J.; Lorente, R.; Munoz-Fernandez, M. A. The Spanish HIV BioBank: a model of cooperative HIV research. 2009, 6, 27.

60. Cohen, J. The immunopathogenesis of sepsis. Nature 2002, 420, 885-91.

61. Consalvi, S.; Biava, M.; Poce, G. COX inhibitors: a patent review (2011-2014). Expert Opin. Ther. Pat. 2015, 25, 1357-1371.

62. Verreck, F. A.; de Boer, T.; Langenberg, D. M.; van der Zanden, L.; Ottenhoff, T. H. Phenotypic and functional profiling of human proinflammatory type-1 and anti-inflammatory type-2 macrophages in response to microbial antigens and IFN-gamma- and CD40L-mediated costimulation. J. Leukoc. Biol. 2006, 79, 285-293.

63. Verreck, F. A.; de Boer, T.; Langenberg, D. M.; Hoeve, M. A.; Kramer, M.; Vaisberg, E.; Kastelein, R.; Kolk, A.; de Waal-Malefyt, R.; Ottenhoff, T. H. Human IL-23-producing type 1 macrophages promote but IL-10-producing type 2 macrophages subvert immunity to (myco)bacteria. Proc. Natl. Acad. Sci. U.S.A. 2004, 101, 4560-4565.

64. Stoeger, T.; Reinhard, C.; Takenaka, S.; Schroeppel, A.; Karg, E.; Ritter, B.; Heyder, J.; Schulz, H. Instillation of six different ultrafine carbon particles indicates a surface area threshold dose for acute lung inflammation in mice. Environ. Health. Perspect. 2006, 114, 328-333.

65. Gordon, S. Alternative activation of macrophages. Nat. Rev. Immunol. 2003, 3, 23-35.

66. Pettus, B. J.; Bielawski, J.; Porcelli, A. M.; Reames, D. L.; Johnson, K. R.; Morrow, J.; Chalfant, C. E.; Obeid, L. M.; Hannun, Y. A. The sphingosine kinase 1/sphingosine-1-phosphate pathway mediates COX-2 induction and PGE2 production in response to TNF-alpha. FASEB J 2003, $17,1411-1421$.

67. Greenhough, A.; Smartt, H. J.; Moore, A. E.; Roberts, H. R.; Williams, A. C.; Paraskeva, C.; Kaidi, A. The COX-2/PGE2 pathway: key roles in the hallmarks of cancer and adaptation to the tumour microenvironment. Carcinogenesis 2009, 30, 377-386.

68. Chen, K. H.; Weng, M. S.; Lin, J. K. Tangeretin suppresses IL-1beta-induced cyclooxygenase (COX)-2 expression through inhibition of p38 MAPK, JNK, and AKT activation in human lung carcinoma cells. Biochem. Pharmacol. 2007, 73, 215-227.

69. Yang, G.; Im, H. J.; Wang, J. H. Repetitive mechanical stretching modulates IL-1beta induced COX-2, MMP-1 expression, and PGE2 production in human patellar tendon fibroblasts. Gene 2005, $363,166-172$.

70. Franciosi, S.; Choi, H. B.; Kim, S. U.; McLarnon, J. G. IL-8 enhancement of amyloid-beta (Abeta 1-42)-induced expression and production of pro-inflammatory cytokines and COX-2 in cultured human microglia. J. Neuroimmunol. 2005, 159, 66-74.

71. Singh, B.; Berry, J. A.; Vincent, L. E.; Lucci, A. Involvement of IL-8 in COX-2-mediated bone metastases from breast cancer. J. Surg. Res. 2006, 134, 44-51.

72. Fong, C. Y.; Pang, L.; Holland, E.; Knox, A. J. TGF-beta1 stimulates IL-8 release, COX-2 expression, and PGE(2) release in human airway smooth muscle cells. Am. J. Physiol. Lung Cell Mol. Physio. 2000, 279, L201-L207.

73. Umesalma, S.; Sudhandiran, G. Differential inhibitory effects of the polyphenol ellagic acid on inflammatory mediators NF-kappaB, iNOS, COX-2, TNF-alpha, and IL-6 in 1,2-dimethylhydrazineinduced rat colon carcinogenesis. Basic Clin. Pharmacol. Toxicol. 2010, 107, 650-5. 
For Graphical Abstract

\section{IMPROVED EFFICIENCY OF IBUPROFEN BY CATIONIC CARBOSILANE DENDRITIC CONJUGATES}

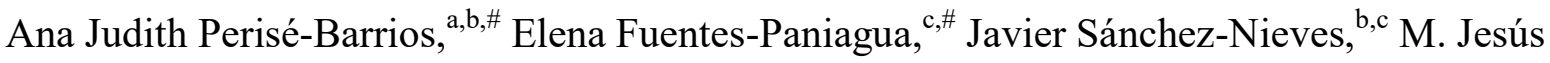
Serramía, ${ }^{a}$ Esther Alonso, ${ }^{a}$ Rosa M. Reguera, ${ }^{\mathrm{d}}$ Rafael Gómez, ${ }^{\mathrm{b}, \mathrm{c}}{ }^{*}$ F. Javier de la Mata, ${ }^{\text {b,c* }}$ and M. Ángeles Muñoz-Fernández ${ }^{\mathrm{a}, \mathrm{b}, *}$

a Laboratorio Inmuno-Biología Molecular, Hospital General Universitario Gregorio Marañón, Spanish HIV HGM BioBank and Instituto de Investigación Sanitaria Gregorio Marañón, 28007 Madrid, Spain. E-mail: mmunoz.hgugm@gmail.com

${ }^{\mathrm{b}}$ Networking Research Center on Bioengineering, Biomaterials and Nanomedicine (CIBERBBN), Spain.

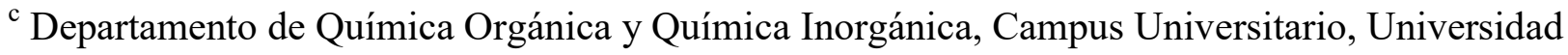
de Alcalá, Alcalá de Henares (Madrid) Spain.E-mail: rafael.gomez@uah.es, javier.delamata@uah.es

${ }^{\mathrm{d}}$ Departamento de Ciencias Biomédicas, Universidad de León, Campus de Vegazana s/n, 24071 León, Spain.

\# Authors contributed equally to this work. 
Ibuprofen-conjugated and non-ibuprofen cationic carbosilane dendritic molecules show important antiinflammatory properties on macrophages.
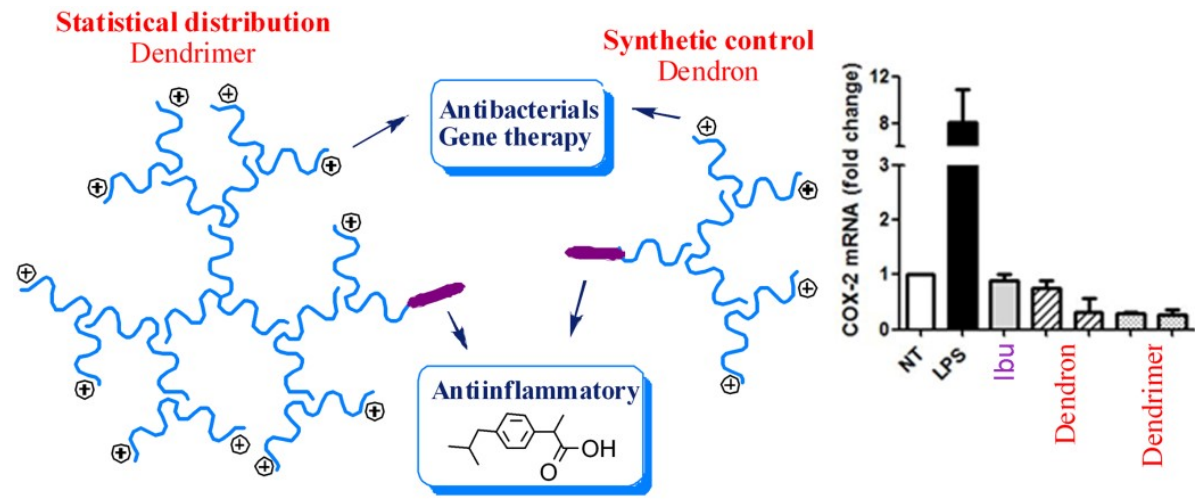